\title{
A New Combination Procedure for the Word Problem that Generalizes Fusion Decidability Results in Modal Logics
}

\author{
Franz Baader ${ }^{\mathrm{a}, 1}$, Silvio Ghilardi ${ }^{\mathrm{b}}$, Cesare Tinelli ${ }^{\mathrm{c}}$ \\ anstitut für Theoretische Informatik, TU Dresden, Germany \\ ${ }^{\mathrm{b}}$ Dipartimento di Scienze dell'Informazione, Università degli Studi di Milano, Italy \\ ${ }^{\mathrm{c}}$ Department of Computer Science, The University of Iowa, USA
}

\begin{abstract}
Previous results for combining decision procedures for the word problem in the nondisjoint case do not apply to equational theories induced by modal logics - which are not disjoint for sharing the theory of Boolean algebras. Conversely, decidability results for the fusion of modal logics are strongly tailored towards the special theories at hand, and thus do not generalize to other types of equational theories.

In this paper, we present a new approach for combining decision procedures for the word problem in the non-disjoint case that applies to equational theories induced by modal logics, but is not restricted to them. The known fusion decidability results for modal logics are instances of our approach. However, even for equational theories induced by modal logics our results are more general since they are not restricted to so-called normal modal logics.
\end{abstract}

\section{Introduction}

The combination of decision procedures for logical theories arises in many areas of logic in computer science, such as constraint solving, automated deduction, term rewriting, modal logics, and description logics. In general, one has two first-order theories $T_{1}$ and $T_{2}$ over the signatures $\Sigma_{1}$ and $\Sigma_{2}$, for which validity of a certain type of formulae (e.g., universal, existential positive, etc.) is decidable. The question is then whether one can combine the decision procedures for $T_{1}$ and $T_{2}$ into one for their union $T_{1} \cup T_{2}$. The problem is usually

1 Partially suported by the German Research Foundation (DFG) under grant BA $1122 / 3-3$. 
much easier, though not at all trivial, if the theories do not share symbols, i.e., if $\Sigma_{1} \cap \Sigma_{2}=\emptyset$. For non-disjoint signatures, the combination of theories can easily lead to undecidability, and thus one must find appropriate restrictions on the theories to be combined.

In automated deduction, the Nelson-Oppen combination procedure [1,2] as well as the problem of combining decision procedures for the word problem [3-7] have drawn considerable attention. The Nelson-Oppen method combines decision procedures for the validity of quantifier-free formulae in so-called stably infinite theories. If we restrict the attention to equational theories, ${ }^{2}$ then it is easy to see that the validity of arbitrary quantifier-free formulae can be reduced to the validity of formulae of the form

$$
s_{1} \approx t_{1} \wedge \ldots \wedge s_{n} \approx t_{n} \rightarrow s \approx t
$$

where $s_{1}, \ldots, t$ are terms. This is an easy consequence of the fact that equational theories are convex [2], i.e., a conjunction of equations implies a disjunction of equations iff it implies one of the disjuncts. Thus, in the case of equational theories the Nelson-Oppen method combines decision procedures for the conditional word problem (i.e., for the validity of conditional equations of the above form).

Though this may at first sight sound surprising, combining decision procedures for the word problem (i.e., for validity of equations $s \approx t$ ) is a harder task: the known combination algorithms for the word problem are more complicated than the Nelson-Oppen method, and the same applies to their proofs of correctness. The reason is that the algorithms for the component theories are then less powerful. For example, if one applies the Nelson-Oppen method to a word problem $s \approx t$, then the method will generate as input for the component procedures conditional word problems, not word problems - see [7] for a more detailed discussion. Both the Nelson-Oppen method and the methods for combining decision procedures for the word problem have been generalized to the non-disjoint case [8-11]. The main restriction on the theories to be combined is that they share only so-called constructors.

In modal logic, one is interested in the question of which properties (such as decidability, interpolation, finite axiomatizability) transfer from component modal logics to their fusion. For the decidability transfer, one usually considers two different decision problems, the validity problem (Is the formula $\varphi$ a theorem of the logic?) and the relativized validity problem (Does the formula $\varphi$ follow from the global assumption $\psi$ ?). There are strong combination results showing that in many cases decidability transfers from two modal logics to their fusion [12-18]. Again, transfer results for the harder decision problem,

$\overline{2}$ Equational theories are stably infinite if one adds the axiom $\exists x, y . x \not \approx$ that prevents trivial, one-element models [7]. 
relativized validity, are easier to show than for the simpler one, validity. ${ }^{3}$ In fact, for validity the results only apply to so-called normal modal logics, ${ }^{4}$ whereas this restriction is not necessary for relativized validity.

There is a close connection between the (conditional) word problem and the (relativized) validity problem in modal logics. In fact, in classical modal logics, which encompass most well-known modal logics, modal formulae can be viewed as terms on which the equivalence of formulae induces an equational theory. The fusion of modal logics then corresponds to the union of the corresponding equational theories, and the (relativized) validity problem to the (conditional) word problem. The union of the equational theories corresponding to two modal logics is over non-disjoint signatures since the Boolean operators are shared. Unfortunately, in this setting the Boolean operators are not shared constructors in the sense of $[9,10]$ (see [11]), and thus the decidability transfer results for fusions of modal logics cannot be obtained as special cases of the results in $[9-11]$.

Recently, a new generalization of the Nelson-Oppen combination method to non-disjoint theories was developed in $[19,20]$. The main restriction on the theories $T_{1}$ and $T_{2}$ to be combined is that they are compatible with their shared theory $T_{0}$, and that their shared theory is locally finite (i.e., its finitely generated models are finite). A theory $T$ is compatible with a theory $T_{0}$ iff

(1) $T_{0} \subseteq T$;

(2) $T_{0}$ has a model completion $T_{0}^{*}$; and

(3) every model of $T$ embeds into a model of $T \cup T_{0}^{*}$.

It is well-known that the theory $B A$ of Boolean algebras is locally finite, and in [20] it is shown that the equational theories induced by modal logics are compatible with $B A$. Thus, the combination method in [20] applies to (equational theories induced by) modal logics. However, since it generalizes the Nelson-Oppen method, it only yields transfer results for the decidability of the conditional word problem (i.e., the relativized validity problem).

In the present paper, we address the harder problem of designing a combination method for the word problem in the non-disjoint case which has the known transfer results for decidability of validity in modal logics as instances. As we will see, our approach strictly generalizes these results since it does not require the modal logics to be normal. The question of whether such transfer results also held for non-normal modal logics was a long-standing open problem in modal logics. In addition to the conditions imposed in [19,20] (i.e.,

3 Relativized validity is indeed a harder problem since in modal logics the deduction theorem typically does not hold.

4 An exception is [17], where only the existence of "covering normal terms" is required. 
compatibility of the component theories with the shared theory $T_{0}$, which is locally finite), our method needs the shared theory $T_{0}$ to have local solvers. Roughly speaking, this is the case if in $T_{0}$ one can solve an arbitrary system of equations with respect to any of its variables. Since this allows one to solve systems of equations by an elimination procedure similar to Gaussian elimination known from linear algebra, we call such theories Gaussian.

In the next section, we introduce some basic notions and results from universal algebra and model theory. In Section 3 we define the restrictions under which our combination approach applies, and give some examples of theories satisfying these restrictions. In Section 4, we describe the new combination procedure, and in Section 5 we show that it is sound and complete. Section 6 shows that the restrictions imposed by our procedure are satisfied by all modal logics where equivalence of formulae induces an equational theory. In particular, we show there that the theory of Boolean algebras is Gaussian. This result is obtained as a consequence of results for unification in Boolean rings [21]. In this section, we also analyze the complexity of our combination procedure if applied to modal logics, and illustrate the working of the procedure on two examples.

\section{Preliminaries}

In this paper we will use standard notions from equational logic, universal algebra and term rewriting (see, e.g., [22]). We consider only first-order theories (with equality $\approx$ ) over a functional signature.

A signature $\Sigma$ is a set of function symbols, each with an associated arity, an integer $n \geq 0$. A constant symbol is a function symbol of zero arity. We use the letters $\Sigma, \Omega$, possibly with subscripts, to denote signatures. Throughout the paper, we fix a countably-infinite set $V$ of variables and a countablyinfinite set $C$ of free constants, both disjoint with any signature $\Sigma .{ }^{5}$ For any $X \subseteq V \cup C, T(\Sigma, X)$ denotes the set of $\Sigma$-terms over $X$, i.e., first-order terms with variables and free constants in $X$ and function symbols in $\Sigma$. First-order $\Sigma$-formulae are defined in the usual way, using equality as the only predicate symbol. A $\Sigma$-sentence is a $\Sigma$-formula without free variables. We use $\perp$ and $\top$ to denote the universally false and the universally true formula, respectively. An equational theory $E$ over $\Sigma$ is a set of (implicitly universally quantified) $\Sigma$-identities of the form $s \approx t$, where $s, t \in T(\Sigma, V)$.

As usual, first-order interpretations of $\Sigma$ are called $\Sigma$-algebras. We denote algebras by calligraphic letters $(\mathcal{A}, \mathcal{B}, \ldots)$, and their carriers by the corre-

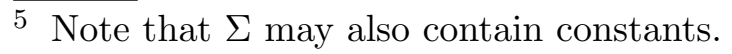


sponding Roman letter $(A, B, \ldots)$. The interpretation of the symbol $f \in \Sigma$ in the $\Sigma$-algebra $\mathcal{A}$ is denoted by $f^{\mathcal{A}}$. A $\Sigma$-algebra $\mathcal{A}$ is a model of a set $\Gamma$ of $\Sigma$-sentences iff it satisfies every sentence in $\Gamma$. For every theory $E$, set $\Gamma$ of sentences and sentence $\varphi$, we write $\Gamma \models_{E} \varphi$ if every model of $E$ that satisfies $\Gamma$ also satisfies $\varphi$. When $\Gamma$ is the empty set, we write just $\models_{E} \varphi$, as usual. We denote by $\approx_{E}$ the equational consequences of $E$, i.e., the relation $\approx_{E}=\left\{(s, t) \in T(\Sigma, V \cup C) \times T(\Sigma, V \cup C) \mid \models_{E} s \approx t\right\}$. The word problem for $E$ is the problem of deciding the relation $\approx_{E}$, that is, deciding for any two terms $s, t \in T(\Sigma, V \cup C)$ whether $s \approx_{E} t$ holds or not. We have defined the word problem for terms including free constants since we will consider such terms later on. Note however that, since free constants behave just like variables in validity problems, the word problem is decidable for terms in $T(\Sigma, V \cup C)$ iff it is decidable for terms in $T(\Sigma, V)$.

Given a $\Sigma$-algebra $\mathcal{A}$ and a subset $G \subseteq A$, the element $a \in A$ is $\Sigma$-generated by $G$ in $\mathcal{A}$ if $a$ can be obtained from $G$ by iterated application of the algebra operations (i.e., the interpretations of the function symbols from $\Sigma$ in $\mathcal{A}$ ). The algebra $\mathcal{A}$ is $\Sigma$-generated by $G$ if all its elements are $\Sigma$-generated by $G$.

A $\Sigma$-algebra $\mathcal{A}$ is called free over the generators $G \subseteq A$ in a class of $\Sigma$-algebras $\mathcal{K}$ iff

- $\mathcal{A}$ is $\Sigma$-generated by $G$;

- $\mathcal{A}$ belongs to $\mathcal{K}$;

- every mapping $f: G \rightarrow B$ from $G$ into the carrier of a $\Sigma$-algebra $\mathcal{B} \in \mathcal{K}$ can be extended to a $\Sigma$-homomorphism $h: \mathcal{A} \rightarrow \mathcal{B}{ }^{6}$

It is easy to see that algebras that are free for the same class over sets of generators of the same cardinality are isomorphic. If the set $G$ is empty, then the free algebra with generators $G$ in $\mathcal{K}$ is also called the initial algebra of $\mathcal{K}$.

Free and initial algebras need not exist for arbitrary classes of $\Sigma$-algebras, but they exist for classes defined by identities. A given equational theory $E$ over $\Sigma$ defines a $\Sigma$-variety, the class of all models of $E$. When $E$ is non-trivial, i.e., has models of cardinality greater than 1 , this variety, also referred to as non-trivial, contains free algebras for any set of generators. We will call these algebras $E$-free algebras. Given a set of generators $X$, the $E$-free algebra with generators $X$ can be obtained as the quotient term algebra $T(\Sigma, X) / \approx_{E}$. In particular, if $s, t \in T(\Sigma, X)$, then the identity $s \approx t$ holds in the $E$-free algebra with generators $X$ iff $\models_{E} s \approx t$.

$\overline{6}$ The concept of a free algebra in a class $\mathcal{K}$ of algebras can be more generally defined by a suitable universal property that does not refer to the notion of generators $[23,24]$. When $\mathcal{K}$ is a non-trivial variety (see later), the definition used in this paperand in most books on universal algebra (see, e.g., [25]) and model theory (e.g., $[26]$ ) - coincides with the one obtained through the universal property. 
In this paper, we often consider several signatures at the same time. If $\Sigma \subseteq \Omega$, then any $\Omega$-algebra can also be viewed as a $\Sigma$-algebra: if $\mathcal{A}$ is an $\Omega$-algebra and $\Sigma \subseteq \Omega$, we denote by $\mathcal{A}^{\Sigma}$ the $\Sigma$-reduct of $\mathcal{A}$, i.e., the algebra obtained from $\mathcal{A}$ by ignoring the symbols in $\Omega \backslash \Sigma$. In this setting, $\mathcal{A}$ is called an expansion of $\mathcal{A}^{\Sigma}$ to the signature $\Omega$.

An embedding of a $\Sigma$-algebra $\mathcal{A}$ into a $\Sigma$-algebra $\mathcal{B}$ is an injective $\Sigma$-homomorphism from $\mathcal{A}$ to $\mathcal{B}$. If such an embedding exists, we say that $\mathcal{A}$ can be embedded into $\mathcal{B}$. The algebra $\mathcal{A}$ is a subalgebra of $\mathcal{B}$ iff $\mathcal{A}$ can be embedded into $\mathcal{B}$ by the inclusion function. It is easy to show that the composition of two embeddings is also an embedding. If $\mathcal{A}$ is $\Sigma$-algebra and $\mathcal{B}$ is an $\Omega$-algebra with $\Sigma \subseteq \Omega$, we say that $\mathcal{A}$ can be $\Sigma$-embedded into $\mathcal{B}$ if there is an embedding of $\mathcal{A}$ into $\mathcal{B}^{\Sigma}$. We call the corresponding embedding a $\Sigma$-embedding of $\mathcal{A}$ into $\mathcal{B}$. If this embedding is the inclusion function, then we say that $\mathcal{A}$ is a $\Sigma$-subalgebra of $\mathcal{B}$.

If $E$ is an equational theory over $\Sigma$ and $X \subseteq Y$ are sets (of generators), then the $E$-free algebra with generators $X$ can be $\Sigma$-embedded into the $E$-free algebra with generators $Y$ by the embedding induced by the inclusion function from $X$ to $Y$. In particular, the initial algebra for $E$ can be $\Sigma$-embedded into any $E$-free algebra.

Given a signature $\Sigma$ and a set $X$ disjoint with $\Sigma$, we denote by $\Sigma(X)$ the signature obtained by adding the elements of $X$ as constant symbols to $\Sigma$. A ground $\Sigma(X)$-literal is a literal over the signature $\Sigma(X)$ not containing variables, i.e., an identity $s \approx t$ or a negated identity $\neg s \approx t$ for terms $s, t \in$ $T(\Sigma, X)$. A ground $\Sigma(X)$-formula is a Boolean combination of ground $\Sigma(X)$ literals. When $X$ is included in the carrier of a $\Sigma$-algebra $\mathcal{A}$, we can view $\mathcal{A}$ as a $\Sigma(X)$-algebra by interpreting each $x \in X$ by itself. If $X$ is a set of generators for $\mathcal{A}$, the $\Sigma$-diagram $\Delta_{X}^{\Sigma}(\mathcal{A})$ of $\mathcal{A}$ (w.r.t. $X$ ) consists of all ground $\Sigma(X)$-literals that hold in $\mathcal{A}$. We write just $\Delta^{\Sigma}(\mathcal{A})$ when $X$ coincides with the whole carrier of $\mathcal{A}$. By a result known as Robinson's Diagram Lemma [27] embeddings and diagrams are related as follows.

Lemma 2.1 Let $\mathcal{A}$ be a $\Sigma$-algebra generated by a set $X$, and let $\mathcal{B}$ be an $\Omega$ algebra for some $\Omega \supseteq \Sigma(X)$. Then $\mathcal{A}$ can be $\Sigma(X)$-embedded into $\mathcal{B}$ iff $\mathcal{B}$ is a model of $\Delta_{X}^{\Sigma}(\mathcal{A})$.

A consequence of the lemma above, which we will use later, is that if two $\Sigma$-algebras $\mathcal{A}, \mathcal{B}$ are both generated by a set $X$ and if one of them, say $\mathcal{B}$, satisfies the other's diagram w.r.t. $X$, then the two algebras are isomorphic: in fact, if one views $\mathcal{A}$ and $\mathcal{B}$ as $\Sigma(X)$-algebras, then " $\mathcal{B}$ satisfies the diagram of $\mathcal{A}$ (w.r.t. $X$ )" implies that there is a $\Sigma(X)$-embedding of $\mathcal{A}$ into $\mathcal{B}$. This embedding maps $X$ to $X$ and, since $X$ generates $\mathcal{B}$, it is surjective, and thus an isomorphism. 
Ground formulae are invariant under embeddings in the following sense.

Lemma 2.2 Let $\mathcal{A}$ be a $\Sigma$-algebra that can be $\Sigma$-embedded into an $\Omega$-algebra $\mathcal{B}$, where $\Sigma \subseteq \Omega$. For all ground $\Sigma(A)$-formulae $\varphi, \mathcal{A}$ satisfies $\varphi$ iff $\mathcal{B}$ satisfies $\varphi$ where $\mathcal{B}$ is extended to an $\Omega(A)$-algebra by interpreting a $\in A$ by its image under the embedding.

When defining our combinability conditions in the next section, we will use the notion of a model completion from model theory. This notion can be defined for arbitrary first-order theories, but here we are interested only in the equational case. Notice however that, even if we start with an equational theory, its model completion is usually not equational.

Definition 2.3 (Model Completion) Let $E$ be an equational $\Sigma$-theory and let $E^{*}$ be a first-order $\Sigma$-theory entailing every identity in $E$. Then $E^{*}$ is a model completion of $E$ iff for every model $\mathcal{A}$ of $E$

(1) $\mathcal{A}$ can be embedded into a model of $E^{*}$, and

(2) $E^{*} \cup \Delta^{\Sigma}(\mathcal{A})$ is a complete $\Sigma(A)$-theory. ${ }^{7}$

One can show that, when it exists, the model completion of a theory is unique [27]. We observe that Condition 2 of Definition 2.3 is always satisfied when the theory $E^{*}$ admits quantifier elimination.

Lemma 2.4 If every $\Sigma$-formula $\varphi(\boldsymbol{x})$ is equivalent modulo the theory $E^{*}$ to some quantifier-free formula $\varphi^{\prime}(\boldsymbol{x})$, then $E^{*} \cup \Delta^{\Sigma}(\mathcal{A})$ is a complete $\Sigma(A)$-theory for every model $\mathcal{A}$ of $E$.

Proof. Let $\mathcal{A}$ be a model of $E$ and assume by contradiction that there is a $\Sigma(A)$-sentence $\varphi$ such that neither $\varphi$ nor $\neg \varphi$ is entailed by $E^{*} \cup \Delta^{\Sigma}(\mathcal{A})$. Then, there are models $\mathcal{A}_{1}, \mathcal{A}_{2}$ of $E^{*} \cup \Delta^{\Sigma}(\mathcal{A})$ such that $\varphi$ is true in $\mathcal{A}_{1}$ and false in $\mathcal{A}_{2}$. By Lemma 2.1, $\mathcal{A}$ can be $\Sigma(A)$-embedded into both $\mathcal{A}_{1}$ and $\mathcal{A}_{2}$. If $E$ admits quantifier elimination, it is easy to see that $\varphi$ is equivalent to a ground $\Sigma(A)$-formula $\varphi^{\prime}$ in every model of $E^{*}$. It follows that $\varphi^{\prime}$ is true in $\mathcal{A}_{1}$ (a model of $E^{*}$ ), hence in $\mathcal{A}$ (by Lemma 2.2), and also in $\mathcal{A}_{2}$ (again by Lemma 2.2). But this contradicts the assumption that $\varphi$ is false in $\mathcal{A}_{2}$ (also a model of $E^{*}$ ).

In this paper we consider theories that are obtained as the union of two theories whose signatures may share some symbols. Robinson's Joint Consistency Lemma [27] provides a general sufficient condition for such unions to be consistent.

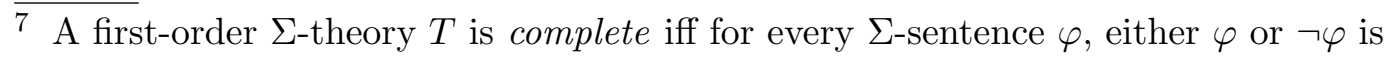
entailed by $T$. 
Lemma 2.5 Let $\Sigma_{1}, \Sigma_{2}$ be signatures and $\Sigma_{0}:=\Sigma_{1} \cap \Sigma_{2}$. Assume that $T_{0}, T_{1}$, $T_{2}$ are first-order theories over the respective signatures $\Sigma_{0}, \Sigma_{1}, \Sigma_{2}$, and $T_{0}$ is complete and contained in both $T_{1}$ and $T_{2}$. Then the consistency of $T_{1}$ and of $T_{2}$ imply the consistency of $T_{1} \cup T_{2}$.

This lemma can be used to show the following result, which will be used in the proof of completeness of our combination procedure.

Lemma 2.6 For $i=1,2$, let $E_{i}$ be an equational theory of signature $\Sigma_{i}$, and assume there is an equational theory $E_{0}$ of signature $\Sigma_{0}=\Sigma_{1} \cap \Sigma_{2}$ with a model completion $E_{0}^{*}$ and such that $\approx_{E_{0}} \subseteq \approx_{E_{i}}$. Let $\mathcal{A}_{i}$ be a model of $E_{i}$ that $\Sigma_{i}$-embeds into a model of $E_{i} \cup E_{0}^{*}$. If $\mathcal{A}_{1}$ and $\mathcal{A}_{2}$ have a common $\Sigma_{0}$-subalgebra $\mathcal{A}_{0}$, then there are a model $\mathcal{A}$ of $E_{1} \cup E_{2}$ and $\Sigma_{i}$-embeddings $f_{i}$ of $\mathcal{A}_{i}$ into $\mathcal{A}$ whose restrictions to $A_{0}$ coincide.

Proof. To simplify the notation, let us assume that $\Sigma_{0}$ contains all the elements of $A_{0}$ as constants, and that $\mathcal{A}_{0}$ interprets each such constant by itself, i.e., $a^{\mathcal{A}_{0}}=a$ for all $a \in A_{0}$. Otherwise we can always add those elements to all the signatures in question. ${ }^{8}$ Let $\mathcal{A}_{0}$ be a $\Sigma_{0}$-subalgebra of $\mathcal{A}_{1}$ and $\mathcal{A}_{2}$, and let $i \in\{1,2\}$.

By assumption, there is a model $\mathcal{B}_{i}$ of $E_{i} \cup E_{0}^{*}$ and a $\Sigma_{i}$-embedding $h_{i}: \mathcal{A}_{i} \longrightarrow$ $\mathcal{B}_{i}$. We can assume without loss of generality that $A_{i}$ is contained in $B_{i}$ and that $h_{i}$ is the inclusion mapping, i.e., $\mathcal{A}_{i}$ is a $\Sigma_{i}$-subalgebra of $\mathcal{B}_{i}$. Otherwise, we could just rename the carrier of $\mathcal{B}_{i}$ appropriately. Let $T_{i}$ be the set of all first-order $\Sigma_{i}\left(B_{i}\right)$-sentences satisfied by $\mathcal{B}_{i}$. We claim that $E_{0}^{*} \cup \Delta^{\Sigma_{0}}\left(\mathcal{A}_{0}\right) \subseteq T_{i}$. The inclusion is immediate for $E_{0}^{*}$ as $\mathcal{B}_{i}$ is a model of $E_{i} \cup E_{0}^{*}$. To see that $\Delta^{\Sigma_{0}}\left(\mathcal{A}_{0}\right) \subseteq T_{i}$, note that $\mathcal{A}_{0}$ is a $\Sigma_{0}$-subalgebra of $\mathcal{B}_{i}$. Since $A_{0} \subseteq \Sigma_{0}$, this implies by Lemma 2.1 that $\mathcal{B}_{i}$ satisfies $\Delta^{\Sigma_{0}}\left(\mathcal{A}_{0}\right)$, hence $\Delta^{\Sigma_{0}}\left(\mathcal{A}_{0}\right) \subseteq T_{i}$.

We have then that $E_{0}^{*} \cup \Delta^{\Sigma_{0}}\left(\mathcal{A}_{0}\right)$, which is a complete theory by Definition 2.3, is included in both $T_{1}$ and $T_{2}$. It follows by Robinson's Joint Consistency Lemma (Lemma 2.5) that $T_{1} \cup T_{2}$ is consistent. Therefore, let $\mathcal{A}$ be any model of $T_{1} \cup T_{2}$ and let $i \in\{1,2\}$. First notice that $\mathcal{A}$ is a model of $E_{1} \cup E_{2}$ as well, because for $i=1,2$, every sentence in $E_{i}$ is satisfied by $\mathcal{B}_{i}$ and so is included in $T_{i}$ by construction. Again by construction of $T_{i}, \mathcal{A}$ satisfies $\Delta^{\Sigma_{i}}\left(\mathcal{B}_{i}\right)$, therefore, by Lemma 2.1 , there is a $\Sigma_{i}\left(B_{i}\right)$-embedding $h_{i}^{\prime}$ of $\mathcal{B}_{i}$ into $\mathcal{A}$. Let $f_{i}$ be the restriction of $h_{i}^{\prime}$ to $A_{i} \subseteq B_{i}$. From the assumption that $\mathcal{A}_{i}$ is a subalgebra of $\mathcal{B}_{i}$ it easily follows that $f_{i}$ is a $\Sigma_{i}$-embedding of $\mathcal{A}_{i}$ into $\mathcal{A}$.

Finally, to see that $f_{1}$ coincides with $f_{2}$ on $A_{0}$, note that for all $a \in A_{0} \subseteq \Sigma_{0}$ we have $f_{1}(a)=f_{1}\left(a^{\mathcal{A}_{0}}\right)=f_{1}\left(a^{\mathcal{B}_{1}}\right)=a^{\mathcal{A}}=f_{2}\left(a^{\mathcal{B}_{2}}\right)=f_{2}\left(a^{\mathcal{A}_{0}}\right)=f_{2}(a)$.

8 This causes no loss of generality because a $\Sigma$-embedding is a $\Sigma^{\prime}$-embedding for all $\Sigma^{\prime} \subseteq \Sigma$. 


\section{The combinability conditions}

Given the equational theories $E_{1}$ and $E_{2}$ to be combined, we want to define conditions under which the decidability of the word problem for $E_{1}$ and $E_{2}$ transfers to their union. We first state the four conditions needed for our transfer result to hold, and then illustrate these conditions by simple examples. The treatment of the main example of this paper, equational theories induced by classical modal logics, is postponed to Section 6 .

\subsection{Defining the conditions}

For the rest of the section we fix two equational theories $E_{1}$ and $E_{2}$ of respective signatures $\Sigma_{1}$ and $\Sigma_{2}$ with a possibly non-empty intersection $\Sigma_{0}$.

Our first condition is that both $E_{1}$ and $E_{2}$ are compatible with a shared subtheory $E_{0}$ over the shared signature $\Sigma_{0}:=\Sigma_{1} \cap \Sigma_{2}$ in the following sense.

Definition 3.1 (Compatibility) Let $E$ be an equational theory over the signature $\Sigma$, and let $E_{0}$ be an equational theory over a subsignature $\Sigma_{0} \subseteq \Sigma$. We say that $E$ is $E_{0}$-compatible iff

(1) $\approx_{E_{0}} \subseteq \approx_{E}$

(2) $E_{0}$ has a model completion $E_{0}^{*}$;

(3) every finitely generated free model of $E$ embeds into a model of $E \cup E_{0}^{*}$.

Some examples of theories that satisfy this definition are discussed in Subsection 3.2 below, in Section 6, and also in $[19,20]$ where a very similar notion of compatibility is introduced for arbitrary first-order theories. When restricted to equational theories, the definition of compatibility in $[19,20]$ is more stringent than the one above because it requires every model of $E$ to embed into a model of $E \cup E_{0}^{*}$, as opposed to just every finitely generated free model. The intuition behind either notion of compatibility is explained in $[19,20]$.

The second condition is that the shared theory $E_{0}$ is locally finite, i.e., all of its finitely generated models are finite. From a more syntactical point of view this means that if $C_{0}$ is a finite subset of $C$ (the set of free constants), then there are only finitely many $E_{0}$-equivalence classes of terms in $T\left(\Sigma_{0}, C_{0}\right)$. For our combination procedure to be effective, we must be able to compute representatives of these equivalence classes.

Definition 3.2 An equational theory $E_{0}$ over the signature $\Sigma_{0}$ is effectively 
locally finite iff for every (finite) tuple $\boldsymbol{c}$ of constants ${ }^{9}$ from $C$ one can effectively compute a finite set of terms $R_{E_{0}}(\boldsymbol{c}) \subseteq T\left(\Sigma_{0}, \boldsymbol{c}\right)$ such that

(1) $s \approx_{E_{0}} t$ for all distinct $s, t \in R_{E_{0}}(\boldsymbol{c})$;

(2) for all terms $s \in T\left(\Sigma_{0}, \boldsymbol{c}\right)$, there is some $t \in R_{E_{0}}(\boldsymbol{c})$ such that $s \approx_{E_{0}} t$.

The third condition on our theories $E_{1}$ and $E_{2}$ is that they are both a conservative extensions of $E_{0}$.

Definition 3.3 Let $E_{0}, E$ be equational theories over the respective signatures $\Sigma_{0}, \Sigma$ where $\Sigma_{0} \subseteq \Sigma$. Then $E$ is a conservative extensions of $E_{0}$ iff

$$
s \approx_{E_{0}} t \quad \text { iff } \quad s \approx_{E} t
$$

for all terms $s, t \in T\left(\Sigma_{0}, V\right)$.

The fourth condition is that the theory $E_{0}$ has local solvers, in the sense that any finite set of equations can be solved with respect to any of its variables. Since this means that finite sets of equations can be solved by something similar to the Gaussian elimination procedure known from linear algebra, we call a theory like that Gaussian.

In the following, we call conjunctions of $\Sigma$-identities e-formulae. We will write $\varphi(\boldsymbol{x})$ to denote an $e$-formula $\varphi$ all of whose variables are included in the tuple $\boldsymbol{x}$. If $\boldsymbol{x}=\left(x_{1}, \ldots, x_{n}\right)$ we will write $\varphi(\boldsymbol{a})$ to denote that $\boldsymbol{a}$ is a tuple of constant symbols of the form $\left(a_{1}, \ldots, a_{n}\right)$ and $\varphi(\boldsymbol{a})$ is the formula obtained from $\varphi$ by replacing every occurrence of $x_{i}$ by $a_{i}$ for $i=1, \ldots, n$.

Definition 3.4 (Gaussian) An equational theory $E_{0}$ is Gaussian iff for every e-formula $\varphi(\boldsymbol{x}, y)$ it is possible to compute an e-formula $C(\boldsymbol{x})$ and a term $s(\boldsymbol{x}, \boldsymbol{z})$ with fresh variables $\boldsymbol{z}$ such that

$$
\models_{E_{0}} \varphi(\boldsymbol{x}, y) \Leftrightarrow(C(\boldsymbol{x}) \wedge \exists \boldsymbol{z} .(y \approx s(\boldsymbol{x}, \boldsymbol{z})))
$$

We call the formula $C$ the solvability condition of $\varphi$ w.r.t. $y$, and the term $s$ $a$ (local) solver of $\varphi$ w.r.t. $y$ in $E_{0}$.

The precise connection between the above definition and Gaussian elimination is explained in Example 3.6 below.

In the next subsection we give examples of theories satisfying even stronger conditions than the combinability conditions introduced above. Let $E_{0}$ and $E$ be equational theories over the respective signatures $\Sigma_{0}$ and $\Sigma$. Then $E$ is called an equational extension of $E_{0}$ iff $\Sigma_{0} \subseteq \Sigma$ and $\approx_{E_{0}} \subseteq \approx_{E}$. Such an

$\overline{9}$ In the following, we will treat tuples also as sets when convenient. 
extension is called trivial iff $E$ is the trivial equational theory, i.e., it has only trivial, one-element models.

Definition 3.5 An equational theory $E_{0}$ is absolutely combinable iff $E_{0}$ is Gaussian and effectively locally finite, and every non-trivial equational extension $E$ of $E_{0}$ is an $E_{0}$-compatible conservative extension of $E_{0}$.

Thus, all the four conditions we introduced above are satisfied by any pair of non-trivial theories $E_{1}, E_{2}$ whose shared theory is absolutely combinable.

\subsection{Examples}

Despite the fact that absolute combinability is a very strong requirement, there are non-artificial examples of theories satisfying it.

Example 3.6 Let $K$ be a fixed field. We consider the theory $T_{K}$ of vector spaces over $K$ whose signature consists of a symbol for addition, a constant 0 for the zero vector, a symbol for the additive inverse and, for every scalar $k \in$ $K$, a unary function symbol $k \cdot(-)$. Axioms are the usual vector spaces axioms (namely, the Abelian group axioms plus the axioms for scalar multiplication).

In this theory, terms are equivalent to linear homogeneous polynomials (with non-zero coefficients) over $K$, i.e., terms of the form

$$
k_{1} \cdot y_{1}+\ldots+k_{n} \cdot y_{n}
$$

where $k_{i} \in K \backslash\{0\}$ and $y_{i}$ is a variable or a free constant. Obviously, this implies that the theory $T_{K}$ is effectively locally finite iff the field $K$ is finite.

Next, we show that $T_{K}$ is Gaussian. Every $e$-formula $\varphi(\boldsymbol{x}, y)$ can be transformed into an equivalent homogeneous system

$$
t_{1}(\boldsymbol{x}, y) \approx 0 \wedge \ldots \wedge t_{k}(\boldsymbol{x}, y) \approx 0
$$

of linear equations with unknowns $\boldsymbol{x}, y$. If $y$ does not occur in $\varphi$, then $\varphi$ is its own solvability condition and $z$ is a local solver. ${ }^{10}$ If $y$ occurs in $\varphi$, then (modulo easy algebraic transformations) we can assume that $\varphi$ contains an equation of the form $y \approx t(\boldsymbol{x})$; this equation gives the local solver, which is $t(\boldsymbol{x})$ (the sequence of existential quantifiers $\exists \boldsymbol{z}$ in (1) is empty), whereas the solvability condition is the $e$-formula obtained from $\varphi$ by eliminating $y$, i.e., replacing $y$ by $t(\boldsymbol{x})$ everywhere in $\varphi$.

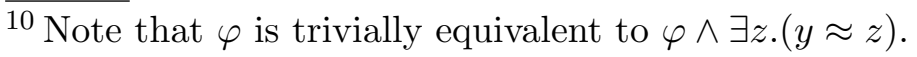


The theory $T_{K}$ admits a model completion $T_{K}^{*}$ whose models are exactly the infinite models of $T_{K} \cdot{ }^{11}$ To see that, it is enough to note that every vector space embeds into an infinite vector space (e.g. into one having an infinite basis), which satisfies the first condition of Definition 2.3. As for the second condition, by Lemma 2.4 it is sufficient to show that $T_{K}^{*}$ admits quantifierelimination. To do that we can consider with no loss of generality only formulae of the form $\exists x . \varphi$, where $\varphi$ is a conjunction of literals each inequivalent to $\perp$ and to $\top$ in the original theory. To eliminate the quantifier $\exists x$ we can proceed as follows. If $\varphi$ contains an identity involving $x$, by solving with respect to $x$ with the usual Gaussian elimination algorithm, we can convert $\varphi$ into a conjunction of the form $x \approx t \wedge \varphi^{\prime}$ where neither $t$ nor $\varphi^{\prime}$ contain $x$. The resulting formula $\exists x .\left(x \approx t \wedge \varphi^{\prime}\right)$, to which $\exists x . \varphi$ is equivalent in the original theory $T_{K}$, is in turn logically equivalent to $\varphi^{\prime}$. If $\varphi$ contains no (positive) identities involving $x$, we can rewrite each negated identity in $\varphi$ containing $x$ into one of the form $x \not z t$, with $x$ not occurring in $t$. The resulting formula, which is equivalent to $\exists x . \varphi$ in $T_{K}$, has the form

$$
\exists x .\left(x \not \approx t_{1} \wedge \cdots \wedge x \not \approx t_{k} \wedge \varphi^{\prime}\right)
$$

where $t_{1}, \ldots, t_{k}$, and $\varphi^{\prime}$ do not contain $x$. This formula is equivalent to $\varphi^{\prime}$ in the extended theory $T_{K}^{*}$ since all the models of that theory are infinite.

It is now very easy to build $T_{K}$-compatible theories. In fact, any non-trivial equational extension $E$ of $T_{K}$ is $T_{K}$-compatible: this is because every finitely generated $E$-free algebra embeds into the countably generated $E$-free algebra, and the latter is always infinite for non-trivial $E$.

Also, notice that, if $E$ is an equational extension of $T_{K}$ that is not conservative, then $E$ is trivial. In fact, if $E$ is a non-conservative extension of $T_{K}$, then it is not difficult to see that there must be a non-zero linear polynomial that is equivalent to zero in $E$, i.e., there is $k \neq 0$ and a polynomial $p(\boldsymbol{x})$ not containing $y$ such that

$$
\models_{E} k \cdot y+p(\boldsymbol{x}) \approx 0 .
$$

Then $\models_{E} y \approx k^{-1} p(\boldsymbol{x})$ and also $\models_{E} y^{\prime} \approx k^{-1} p(\boldsymbol{x})$ (by renaming $y$ into $y^{\prime}$ ), which shows that $\models_{E} y \approx y^{\prime}$, i.e. $E$ is trivial.

Thus, we have shown that the theory $T_{K}$ of vector spaces over the field $K$ is absolutely combinable if $K$ is finite.

Example 3.7 Another example, which is very similar to the one above, is the pure equality theory $E_{=}$, that is, the empty theory in the empty signature.

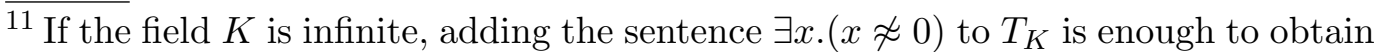
$T_{K}^{*}$. Otherwise, it is enough to add for each $n>0$ a sentence satisfied in exactly all models of cardinality at least $n$.
} 
This theory is Gaussian: to show this, one can argue as in the previous example. Specifically, let $\varphi(\boldsymbol{x}, y)$ be an $e$-formula: if $\varphi$ contains an equation like $y \approx x_{i}$, then $x_{i}$ is the local solver and the solvability condition is obtained by replacing $y$ by $x_{i}$ in $\varphi$. Otherwise, we first remove the trivial equations $y \approx y$; at this point, $\varphi(\boldsymbol{x}, y)$ does not contain $y$ anymore, so it is its own solvability condition (the solvability condition reduces to the tautology $T$ if no equation survives); the local solver is clearly $z$.

$E_{=}$admits the theory $E_{=}^{*}$ of an infinite set as a model completion: in fact, by an argument very similar to the one in the previous example, it is easy to show that $E_{=}^{*}$ has quantifier elimination and that every model of $E$ (i.e., every set) can be embedded into a model of $E_{=}^{*}$ (i.e., into an infinite set). That $E_{=}$ is effectively locally finite is also clear.

Take now any non-trivial equational theory $E$. It is immediate that, for being non-trivial, $E$ is a conservative extension of $E_{=}$. We show that $E$ is also $E_{=-}$ compatible. Now, points 1 and 2 of Definition 3.1-requiring that $\approx_{E=} \subseteq \approx_{E}$ and $E_{=}$admits a model completion - are immediately satisfied as already explained. Point 3 -requiring that every free model of $E$ with finitely many generators be embeddable in a model of $E \cup E_{=}^{*}$ - is satisfied because every finitely generated free model of an equational theory $E$ embeds into an infinitely generated free model of $E$. Since the latter model is infinite for non-trivial $E$, it is also a model of $E \cup E_{=}^{*}$.

Thus, we have shown that the pure equality theory $E_{=}$is absolutely combinable.

In Section 6, we will prove the remarkable fact that the theory of Boolean algebras is absolutely combinable as well. In particular, we will show that the theory of Boolean algebras is Gaussian. This is a more sophisticated example of a Gaussian theory, in which the string of existential quantifiers $\exists \boldsymbol{z}$ in (1) can be both not empty and applied to a non-trivial solver. ${ }^{12}$

Next, we give an example of a theory that is not Gaussian.

Example 3.8 Let $\Sigma$ be a signature consisting of a single unary function symbol $f$, and let $E_{\emptyset}$ be the empty theory over this signature. This theory is not Gaussian. In fact, consider the $e$-formula $y \approx f(y)$, and assume that it has a local solver $s$ and a solvability condition $C$ such that

$$
\models_{E_{\emptyset}} y \approx f(y) \Leftrightarrow(C \wedge \exists \boldsymbol{z} .(y \approx s(\boldsymbol{z}))) .
$$

Since $C$ does not contain variables and there are no variable-free $\Sigma$-terms, $C$

\footnotetext{
${ }^{12}$ Note that in the above examples, we always have that either there are no parameters $\boldsymbol{z}$, or that the solver is the trivial term $z$ for a new variable $z$.
} 
must be the empty conjunction, which is trivially valid in $E_{\emptyset}$. Since $\Sigma$ contains only the unary function symbol $f$, the solver $s$ must be of the form $s=f^{k}(z)$ for some $k \geq 0$ and a variable $z$ different from $y$. Thus, we have

$$
\models_{E_{\emptyset}} y \approx f(y) \Leftrightarrow \exists z \cdot\left(y \approx f^{k}(z)\right)
$$

However, this equivalence does not hold in $E_{\emptyset}$. For example, consider the model whose domain are the natural numbers and where $f$ is interpreted as the successor function. For $y=k$ there exists a $z$ (namely, $z=0$ ) such that $k=f^{k}(z)=z+k$. However, $k \neq k+1=f(k)$.

We recall that an equational theory $E$ has unitary unification type iff every solvable unification problem has a most general $E$-unifier (see, e.g., [28]). The last example shows that not every theory with unitary unification type is Gaussian. Even if we will not need this result in the rest of the paper it is interesting to point out the opposite inclusion does hold. We show that in the following, using basic notions from unification theory (as, e.g., introduced in $[28])$.

Lemma 3.9 Every Gaussian equational theory has a unitary unification type.

Proof. Let $E$ be a Gaussian equational theory. Since $E$-unification problems are sets of equations, they can be viewed as $e$-formulae. Thus, let us consider the unification problem $\varphi(\boldsymbol{x})$, where $\varphi$ is an $e$-formula. We recall that a solution of this problem, also called a $E$-unifier, is a substitution $\sigma$ (i.e., a replacement of the variables $\boldsymbol{x}$ by terms) such that $\models_{E} \varphi \sigma .{ }^{13} E$-unifiers are compared with respect to instantiation modulo $E$ on the variables $\boldsymbol{x}$ occurring in $\varphi$ : a substitution $\mu$ is more general than a substitution $\sigma$ w.r.t. $\boldsymbol{x}\left(\mu \leq_{E}^{\boldsymbol{x}} \sigma\right)$ iff there is a substitution $\lambda$ such that $\models_{E} x \sigma \approx x \mu \lambda$ for all variables $x$ in $\boldsymbol{x}$. We show that every solvable $E$-unification problem $\varphi(\boldsymbol{x})$ has a most general $E$-unifier (mgu), i.e., an $E$-unifier $\mu$ such that $\mu \leq_{E}^{\boldsymbol{x}} \sigma$ for all $E$-unifiers $\sigma$ of $\varphi(\boldsymbol{x})$.

Assume that $\varphi(\boldsymbol{x})$ has a solution. Since $E$ is Gaussian, we can successively eliminate all the variables $\boldsymbol{x}=x_{1}, \ldots, x_{n}$ from $\varphi$ and obtain that

$$
\models_{E} \varphi(\boldsymbol{x}) \Leftrightarrow C \wedge \exists \boldsymbol{z} \cdot\left(\bigwedge_{i=1}^{n} x_{i} \approx s_{i}(\boldsymbol{z})\right),
$$

where $C$ is a ground $e$-formula and the $s_{i}$ are terms containing only variables from the tuple of fresh variables $\boldsymbol{z}$. From (2) and the fact that $\varphi(\boldsymbol{x})$ has a solution it follows that $E \models C$. This means that (2) can be restated as

$$
\models_{E} \varphi(\boldsymbol{x}) \Leftrightarrow \exists \boldsymbol{z} \cdot\left(\bigwedge_{i=1}^{n} x_{i} \approx s_{i}(\boldsymbol{z})\right) .
$$

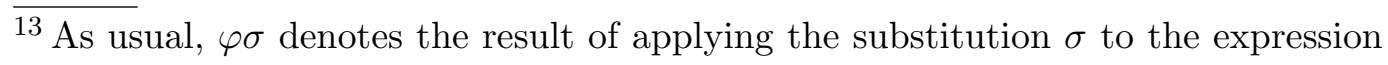
$\varphi$. 
We claim that the substitution

$$
\mu:=\left\{x_{1} \mapsto s_{1}(\boldsymbol{z}), \ldots, x_{n} \mapsto s_{n}(\boldsymbol{z})\right\}
$$

is an mgu of $\varphi(\boldsymbol{x})$. To see that $\mu$ is an $E$-unifier of $\varphi(\boldsymbol{x})$ observe that the right to left implication of (3) is equivalent to

$$
\models_{E} \bigwedge_{i=1}^{n} x_{i} \approx s_{i}(\boldsymbol{z}) \Rightarrow \varphi(\boldsymbol{x})
$$

which obviously implies that $\models_{E} \varphi \mu$. To see that $\mu$ is most general w.r.t. $\boldsymbol{x}$, we must show that it is more general than any other unifier $\sigma$. Thus, assume that $\sigma$ is a unifier of $\varphi(\boldsymbol{x})$. This means that $\models_{E} \varphi \sigma$, and thus we have by (3) that

$$
\models_{E} \exists \boldsymbol{z} \cdot \bigwedge_{i=1}^{n} x_{i} \sigma \approx s_{i}(\boldsymbol{z}),
$$

that is, the formula $\psi:=\exists \boldsymbol{z} . \bigwedge_{i=1}^{n} x_{i} \sigma \approx s_{i}(\boldsymbol{z})$ holds in all models of $E$. In particular, $\psi$ holds in the $E$-free algebra with a countably infinite set of generators. The elements of this algebra are $\approx_{E^{-}}$equivalence classes of terms over the countably infinite set $X$ of all variables. Thus, we obtain that

$$
\models_{E} \bigwedge_{i=1}^{n} x_{i} \sigma \approx s_{i}(\boldsymbol{t})
$$

for some tuple of terms $\boldsymbol{t}$. Let $\lambda$ be the substitution that maps every element of $\boldsymbol{z}$ to the corresponding element of $\boldsymbol{t}$. Then we have that $\models_{E} x_{i} \sigma \approx x_{i} \mu \lambda$ for $i=1, \ldots, n$. This shows that $\mu \leq_{E}^{\boldsymbol{x}} \sigma$, making $\mu$ a most general $E$-unifier of $\varphi(\boldsymbol{x})$.

\section{The combination procedure}

In this section we describe an algorithm for combining two procedures deciding the world problem in two theories $E_{1}$ and $E_{2}$, respectively, into a procedure deciding the world problem in $E_{1} \cup E_{2}$. For that we assume that $E_{1}, E_{2}$ are equational theories over the signatures $\Sigma_{1}, \Sigma_{2}$ with decidable word problems, and that there exists an equational theory $E_{0}$ over the signature $\Sigma_{0}:=\Sigma_{1} \cap \Sigma_{2}$ such that

- $E_{0}$ is Gaussian and effectively locally finite;

- for $i=1,2, E_{i}$ is $E_{0}$-compatible and a conservative extension of $E_{0}$. 


\subsection{Abstraction rewrite systems}

Our combination procedure works on the following data structure.

Definition 4.1 An abstraction rewrite system (ARS) $R$ is a finite ground rewrite system that can be partitioned into two subsets $R_{1}$ and $R_{2}$ such that

- for $i=1,2$, the rules of $R_{i}$ are of the form $a \rightarrow t$ where $a \in C$ and $t \in T\left(\Sigma_{i}, C\right)$, and every constant a occurs at most once as a left-hand side in $R_{i}$;

- $R=R_{1} \cup R_{2}$ is terminating.

The $A R S R$ is an initial ARS iff every constant occurs at most once as a left-hand side in the whole $R$.

Since every ARS $R$ is terminating, we can find a strict total ordering $>$ on the left-hand side constants of $R$ such that for all $a \rightarrow t \in R$, the term $t$ contains only left-hand side constants smaller than $a$. In particular, for $i=1,2, R_{i}$ is also terminating, and the restriction that every constant occurs at most once as a left-hand side in $R_{i}$ implies that $R_{i}$ is confluent. We denote the unique normal form of a term $s$ w.r.t. $R_{i}$ by $s \downarrow_{R_{i}}$.

Given a ground rewrite system $R$, an equational theory $E$, and an $e$-formula $\psi$, we write $R \models_{E} \psi$ to express that $\{l \approx r \mid l \rightarrow r \in R\} \models_{E} \psi$.

The following results about ARS's will be used several times in the rest of the paper.

Lemma 4.2 Consider an $A R S R=R_{1} \cup R_{2}$ and let $i \in\{1,2\}$. Let a collect the left-hand side constants of $R_{i}$ and $\boldsymbol{c}$ collect the remaining free constants of $R_{i}$. For every $\Sigma_{i}(\boldsymbol{c})$-model $\mathcal{A}$ of $E_{i}$ the following holds:

(1) $\mathcal{A}$ can be expanded to a $\Sigma_{i}(\boldsymbol{a}, \boldsymbol{c})$-model $\widehat{\mathcal{A}}$ of $E_{i}$ that satisfies $R_{i}$.

(2) If $\mathcal{A}$ is an initial $\Sigma_{i}(\boldsymbol{c})$-model of $E_{i}$, then its expansion $\hat{\mathcal{A}}$ is an initial $\Sigma_{i}(\boldsymbol{a}, \boldsymbol{c})$-model of $E_{i} \cup\left\{a \approx t \mid a \rightarrow t \in R_{i}\right\}$.

Proof. Let $a_{n}>a_{n-1}>\cdots>a_{1}$ be a total ordering of the left-hand side (lhs) constants of $R_{i}=\left\{a_{j} \rightarrow t_{j} \mid j=1, \ldots, n\right\}$ such that $t_{j}$ contains only lhs constants smaller than $a_{j}$. Let $\mathcal{A}$ be a $\Sigma_{i}(\boldsymbol{c})$-model of $E_{i}$.

(1) We define expansions $\mathcal{A}_{j}$ of $\mathcal{A}$ that interpret the lhs constants $a_{1}, \ldots, a_{j}$ by induction on $j=0, \ldots, n$ :

- The algebra $\mathcal{A}_{0}$ is defined simply as $\mathcal{A}$.

- For $j>0$, the algebra $\mathcal{A}_{j}$ expands $\mathcal{A}_{j-1}$ by interpreting $a_{j}$ by the interpre- 
tation of $t_{j}$ in $\mathcal{A}_{j-1}$, i.e., $a_{j}^{\mathcal{A}_{j}}:=t_{j}^{\mathcal{A}_{j-1}}$. Note that $t_{j}^{\mathcal{A}_{j-1}}$ is well-defined since $t_{j}$ does not contain any of the constants $a_{j}, \ldots, a_{n}$.

Now, let $\widehat{\mathcal{A}}$ be $\mathcal{A}_{n}$. It is easy to see that this algebra is a $\Sigma_{i}(\boldsymbol{a}, \boldsymbol{c})$-model of $E_{i} \cup\left\{a_{j} \approx t_{j} \mid j=1, \ldots, n\right\}$.

(2) Assume $\mathcal{A}$ is an initial model of $E_{i}$. Since $\mathcal{A}$ is $\Sigma_{i}(\boldsymbol{c})$-generated by $\emptyset$ and the carrier of $\widehat{\mathcal{A}}$ coincides with the carrier of $\mathcal{A}$, the expansion $\widehat{\mathcal{A}}$ is obviously $\Sigma_{i}(\boldsymbol{a}, \boldsymbol{c})$-generated by $\emptyset$. By $(1), \widehat{\mathcal{A}}$ is a model of $E_{i} \cup\left\{a_{j} \approx t_{j} \mid j=1, \ldots, n\right\}$.

To show that $\widehat{\mathcal{A}}$ is initial, assume that the algebra $\mathcal{B}$ is a $\Sigma_{i}(\boldsymbol{a}, \boldsymbol{c})$-model of $E_{i} \cup\left\{a_{j} \approx t_{j} \mid j=1, \ldots, n\right\}$. It is enough to show that there exists a $\Sigma_{i}(\boldsymbol{a}, \boldsymbol{c})$ homomorphism from $\widehat{\mathcal{A}}$ to $\mathcal{B}$. The reduct $\mathcal{B}^{\Sigma_{i}(\boldsymbol{c})}$ is a model of $E_{i}$, and thus there is a $\Sigma_{i}(\boldsymbol{c})$-homomorphism $h: \mathcal{A} \rightarrow \mathcal{B}^{\Sigma_{i}(\boldsymbol{c})}$. We claim that $h$ is also a $\Sigma_{i}(\boldsymbol{a}, \boldsymbol{c})$-homomorphism from $\widehat{\mathcal{A}}$ to $\mathcal{B}$, i.e., $h\left(a_{j}^{\widehat{\mathcal{A}}}\right)=a_{j}^{\mathcal{B}}$ for all $j=1, \ldots, n$. This can be proved by induction on $j$ :

- For $j=1$, we have $h\left(a_{1}^{\widehat{\mathcal{A}}}\right)=h\left(t_{1}^{\mathcal{A}}\right)=t_{1}^{\mathcal{B}}=a_{1}^{\mathcal{B}}$, where the first identity holds by the definition of $\widehat{\mathcal{A}}$, the second since $h$ is a $\Sigma_{i}(\boldsymbol{c})$-homomorphism, and the third since $\mathcal{B}$ satisfies $a_{1} \approx t_{1}$.

- For $j>1$, we have $h\left(a_{j}^{\widehat{\mathcal{A}}}\right)=h\left(t_{j}^{\mathcal{A}}\right)=t_{j}^{\mathcal{B}}=a_{j}^{\mathcal{B}}$, where the first identity holds by the definition of $\hat{\mathcal{A}}$, the second since we know by induction that $h$ is a $\Sigma_{i}\left(a_{1}, \ldots, a_{j-1}, \boldsymbol{c}\right)$-homomorphism, and the third since $\mathcal{B}$ satisfies $a_{j} \approx t_{j}$.

Lemma 4.3 Let $R=R_{1} \cup R_{2}$ be an $A R S$, and $s, t \in T\left(\Sigma_{i}, C\right)$ for some $i \in\{1,2\}$. Then $R_{i} \models_{E_{i}} s \approx t$ iff $s \downarrow_{R_{i}} \approx_{E_{i}} t \downarrow_{R_{i}}$.

Proof. Let $i \in\{1,2\}$.

$(\Leftarrow)$ Obviously, $s \downarrow_{R_{i}} \approx_{E_{i}} t \downarrow_{R_{i}}$ implies $R_{i} \models_{E_{i}} s \approx t$.

$(\Rightarrow)$ Assume that $R_{i} \models_{E_{i}} s \approx t$. Since $R_{i} \models_{E_{i}} s \approx s \downarrow_{R_{i}}$ and $R_{i} \models_{E_{i}} t \approx t \downarrow_{R_{i}}$, this yields $R_{i} \models_{E_{i}} s \downarrow_{R_{i}} \approx t \downarrow_{R_{i}}$. Now assume that $s \downarrow_{R_{i}} \not \varpi_{E_{i}} t \downarrow_{R_{i}}$, i.e., there is a model $\mathcal{A}$ of $E_{i}$ in which the identity $s \downarrow_{R_{i}} \approx t \downarrow_{R_{i}}$ does not hold. Since the terms $s \downarrow_{R_{i}}, t \downarrow_{R_{i}}$ do not contain the left-hand side constants of $R_{i}$, we may assume that $\mathcal{A}$ does not interpret these constants. By Lemma 4.2, we can expand $\mathcal{A}$ to a model $\widehat{\mathcal{A}}$ of $E_{i}$ that also interprets these constants and satisfies $R_{i}$. Since the interpretation of the terms $s \downarrow_{R_{i}}, t \downarrow_{R_{i}}$ in $\widehat{\mathcal{A}}$ coincides with the one in $\mathcal{A}$, this implies that $R_{i} \not \models_{E_{i}} s \downarrow_{R_{i}} \approx t \downarrow_{R_{i}}$, a contradiction.

If we want to decide the word problem in $E_{1} \cup E_{2}$, it is sufficient to consider ground terms with free constants, i.e., terms $s, t \in T\left(\Sigma_{1} \cup \Sigma_{2}, C\right)$. Given such terms $s, t$ we can employ the usual abstraction procedures that replace subterms by new constants in $C$ (see, e.g., [10]) to generate terms $u, v \in$ 
Input: an initial ARS $R=R_{1} \cup R_{2}=\left\{a_{i} \rightarrow t_{i} \mid i=1, \ldots, n\right\}$ and terms $u, v \in T\left(\Sigma_{0}, C\right)$.

Let $\boldsymbol{c}$ collect the free constants in $R, u$, and $v$ that are not in $\left\{a_{1}, \ldots, a_{n}\right\}$. for $i=1$ to $n$ do

1. Let $j$ be such that $a_{i} \rightarrow t_{i} \in R_{j}$ and $k$ such that $\{j, k\}=\{1,2\}$.

2. Let $T=R_{E_{0}}\left(\boldsymbol{a}_{i}, \boldsymbol{c}\right)$ (see Definition 3.2).

3. For each pair of distinct terms $t, t^{\prime} \in T$, test whether $R_{j}^{(i)} \models{ }_{E_{j}} t \approx t^{\prime}$.

4. Let $\varphi\left(\boldsymbol{a}_{i}, \boldsymbol{c}\right)$ be the conjunction of those identities $t \approx t^{\prime}$ for which the test succeeds.

5. Let $s\left(\boldsymbol{a}_{i-1}, \boldsymbol{c}, \boldsymbol{d}\right)$ be a local solver of $\varphi$ w.r.t. $a_{i}$ in $E_{0}$.

6 . Add to $R_{k}$ the new rule $a_{i} \rightarrow s\left(\boldsymbol{a}_{i-1}, \boldsymbol{c}, \boldsymbol{d}\right)$.

done

Output: "yes" if $R_{1}=_{E_{1}} u \approx v$, and "no" otherwise.

Fig. 1. The combination procedure.

$T\left(\Sigma_{0}, C\right)$ and an initial ARS $R=R_{1} \cup R_{2}$ such that

$$
s \approx_{E_{1} \cup E_{2}} t \quad \text { iff } \quad R={ }_{E_{1} \cup E_{2}} u \approx v .
$$

For example, assume that $\Sigma_{1}=\{f, g\}$ and $\Sigma_{2}=\{f, h\}$, and consider the terms $s=f\left(h\left(c_{1}\right), g\left(h\left(c_{1}\right)\right)\right)$ and $t=g\left(f\left(h\left(c_{1}\right), c_{2}\right)\right)$. Then we can take $u=f\left(a_{1}, a_{2}\right)$, $v=a_{3}, R_{1}=\left\{a_{2} \rightarrow g\left(a_{1}\right), a_{3} \rightarrow g\left(f\left(a_{1}, c_{2}\right)\right)\right\}$, and $R_{1}=\left\{a_{1} \rightarrow h\left(c_{1}\right)\right\}$.

Thus, to decide the word problem in $E_{1} \cup E_{2}$, it is sufficient to devise a procedure that can solve problems of the form " $R \models_{E_{1} \cup E_{2}} u \approx v$ ?" where $R$ is an initial ARS and $u, v \in T\left(\Sigma_{0}, C\right)$. We present this procedure next.

\subsection{The combination procedure}

The input of the procedure is an initial ARS $R=R_{1} \cup R_{2}$ and two terms $u, v \in T\left(\Sigma_{0}, C\right)$. Let $>$ be a total ordering of the left-hand side (lhs) constants of $R$ such that for all $a \rightarrow t \in R, t$ contains only lhs constants smaller than $a$. Given this ordering, we can assume that $R=\left\{a_{i} \rightarrow t_{i} \mid i=1, \ldots, n\right\}$ for some $n \geq 0$ where $a_{n}>a_{n-1}>\cdots>a_{1}$.

Note that $u, v$ and each $t_{i}$ may also contain free constants from $C$ that are not left-hand side constants. In the following, we use $\boldsymbol{c}$ to denote a tuple of all these constants. Furthermore, for $j=1,2$ and $i=0, \ldots, n$, we denote by $R_{j}^{(i)}$ the restriction of $R_{j}$ to the rules whose left-hand sides are smaller than or equal to $a_{i}$-where, by convention, $R_{j}^{(0)}$ is the empty system. Finally, for $i=1, \ldots, n$, we denote by $\boldsymbol{a}_{i}$ the tuple $\left(a_{1}, \ldots, a_{i}\right)$. 
The combination procedure is described in Figure 1. Similarly to previous combination procedures for the word problem, the procedure works in essence by incrementally propagating from one component decision procedure to the other entailed identities between ground terms in the shared signature. At each step $i$ of the procedure's main loop, the propagated information is the one expressed by the identities in the formula $\varphi\left(\boldsymbol{a}_{i}, \boldsymbol{c}\right)$, recognized by the decision procedure for $E_{j}$ as consequences of $R_{j}^{(i)}$. The main difference with previous combination methods is that this information is first distilled, so to speak, into a single identity- $a_{i} \approx s\left(\boldsymbol{a}_{i-1}, \boldsymbol{c}, \boldsymbol{d}\right)$ - obtained by solving $\varphi\left(\boldsymbol{a}_{i}, \boldsymbol{c}\right)$ w.r.t. $a_{i}$. This is possible precisely because the shared theory $E_{0}$ is Gaussian. The propagation process is incremental in that at each step $i$ the procedure considers for propagation only equational consequences of $R_{j}^{(i)}$ in the signature $\Sigma_{0}\left(\boldsymbol{a}_{i}, \boldsymbol{c}\right)$, as opposed to equational consequences of the whole $R_{j}$ in the full shared signature $\Sigma_{0}\left(\boldsymbol{a}_{n}, \boldsymbol{c}\right)$.

We point out that all of the steps of the procedure are effective. In fact, Step 1 of the for loop is trivially effective; Step 2 is effective because $E_{0}$ is effectively locally finite by assumption. Step 3 is effective because the test that $R_{j}^{(i)} \models_{E_{j}} t \approx t^{\prime}$ can be reduced by Lemma 4.3 to testing that $t \downarrow_{R_{j}^{(i)}} \approx_{E_{j}} t^{\prime} \downarrow_{R_{j}^{(i)}}$. The latter test is effective because, (i) the word problem in $E_{j}$ is decidable by assumption and (ii) $R_{j}^{(i)}$ is confluent and terminating at each iteration of the loop. Now, in Step 4 the formula $\varphi$ can be computed because $T$ is finite and the local solver in Step 5 can be computed by the algorithm provided by the definition of a Gaussian theory. Step 6 is trivial and for the final test after the loop, the same observations as for Step 3 apply.

A few more remarks on the procedure are in order. In the fifth step of the loop, $\boldsymbol{d}$ is a tuple of new constants introduced by the solver $s$. In the definition of a local solver, we have used variables instead of constants, but this difference will turn out to be irrelevant since free constants behave like variables. One may wonder why the procedure ignores the solvability condition for the local solver. The reason is that this condition follows from both $R_{1}$ and $R_{2}$, as will be shown in the proof of completeness.

Adding the new rule to $R_{k}$ in the sixth step of the loop does not destroy the property of $R_{1} \cup R_{2}$ being an ARS - although it will make it non-initial. In fact, $s\left(\boldsymbol{a}_{i-1}, \boldsymbol{c}, \boldsymbol{d}\right)$ contains only lhs constants smaller than $a_{i}$, and $R_{k}$ before did not contain a rule with lhs $a_{i}$ because the input was an initial ARS.

The test after the loop is performed using $R_{1}, E_{1}$. The choice $R_{1}$ and $E_{1}$ versus $R_{2}$ and $E_{2}$ is arbitrary. As it will be made clear by the completeness proof for the procedure, using $R_{2}, E_{2}$ instead would produce the same results.

Before proving the correctness of the procedure, we illustrate it by a simple example. While the example is restricted to the well studied case of signature- 
disjoint theories, it is enough to show the essence of the propagation mechanism implemented by our procedure.

Example 4.4 Let $E_{1}:=\{f(x, y) \approx f(y, x)\}$ and $E_{2}:=\{g(x, x) \approx x\}$. Its is easy to see that both theories are non-trivial and have decidable word problems. Since these two theories do not share any function symbols, we can use the theory $E_{=}$from Example 3.7 as the shared theory. As argued in that example, $E_{1}, E_{2}$, and $E_{=}$satisfy all of our combinability conditions. Assume then that we want to use our procedure to show whether

$$
\models_{E_{1} \cup E_{2}} g(f(x, y), f(y, g(x, x))) \approx f(x, y)
$$

After the abstraction process, we get the rewrite systems:

$$
\begin{aligned}
& R_{1}=\left\{a_{4} \rightarrow f\left(c_{1}, c_{2}\right), a_{3} \rightarrow f\left(c_{2}, a_{1}\right), a_{2} \rightarrow f\left(c_{1}, c_{2}\right)\right\} \text { and } \\
& R_{2}=\left\{a_{5} \rightarrow g\left(a_{2}, a_{3}\right), a_{1} \rightarrow g\left(c_{1}, c_{1}\right)\right\}
\end{aligned}
$$

and the goal identity

$$
a_{5} \approx a_{4}
$$

where $a_{1}, \ldots, a_{5}, c_{1}$ and $c_{2}$ are fresh constants, with $c_{1}$ and $c_{2}$ replacing the variables $x$ and $y$, respectively.

During the first execution of the procedure's loop, the procedure considers the lhs constant $a_{1}$ and the free constants $c_{1}, c_{2}$. Since the signature of $E_{=}$ is empty, these are also the terms to be considered for $R_{E_{0}}\left(a_{1}, c_{1}, c_{2}\right)$. The identity $a_{1} \approx c_{1}$ is the only identity between distinct terms of $R_{E_{0}}\left(a_{1}, c_{1}, c_{2}\right)$ for which the test in Step 3 of the procedure succeeds. Using the procedure described in Example 3.7, Step 5 computes the solver $s=c_{1}$ for the formula $\varphi\left(a_{1}, c_{1}, c_{2}\right)=a_{1} \approx c_{1}$. Hence, Step 5 adds the rule $a_{1} \rightarrow c_{1}$ to $R_{1}$ yielding the new systems:

$$
\begin{aligned}
& R_{1}=\left\{a_{4} \rightarrow f\left(c_{1}, c_{2}\right), a_{3} \rightarrow f\left(c_{2}, a_{1}\right), a_{2} \rightarrow f\left(c_{1}, c_{2}\right), \underline{a_{1} \rightarrow c_{1}}\right\} \\
& R_{2}=\left\{a_{5} \rightarrow g\left(a_{2}, a_{3}\right), a_{1} \rightarrow g\left(c_{1}, c_{1}\right)\right\}
\end{aligned}
$$

The second iteration of the loop considers the constants $a_{1}, a_{2}$ and $c_{1}, c_{2}$. The only non-trivial identity involving the terms $a_{1}, a_{2}, c_{1}, c_{2}$ that is entailed by $R_{1}^{(2)}$ in $E_{1}$ is $a_{1} \approx c_{1}$. Solving $\varphi\left(a_{1}, a_{2}, c_{1}, c_{2}\right)=a_{1} \approx c_{1}$ w.r.t. $a_{2}$ using the procedure described in Example 3.7 produces the solver $s=d_{1}$, where $d_{1}$ is a fresh constant. Consequently, Step 6 adds the rewrite rule $a_{2} \rightarrow d_{1}$ to $R_{2}$, yielding the new systems:

$$
\begin{aligned}
& R_{1}=\left\{a_{4} \rightarrow f\left(c_{1}, c_{2}\right), a_{3} \rightarrow f\left(c_{2}, a_{1}\right), a_{2} \rightarrow f\left(c_{1}, c_{2}\right), a_{1} \rightarrow c_{1}\right\} \\
& R_{2}=\left\{a_{5} \rightarrow g\left(a_{2}, a_{3}\right), \underline{a_{2} \rightarrow d_{1}}, a_{1} \rightarrow g\left(c_{1}, c_{1}\right)\right\}
\end{aligned}
$$


The third iteration of the loop considers the constants $a_{1}, a_{2}, a_{3}$ and $c_{1}, c_{2}$. The only relevant new identity (i.e., non-trivial and involving $a_{3}$ ) that can now be derived in Step 3 is $a_{3} \approx a_{2}$, which leads to the solver $s=a_{2}$ w.r.t. $a_{3}$ and the new systems:

$$
\begin{aligned}
& R_{1}=\left\{a_{4} \rightarrow f\left(c_{1}, c_{2}\right), a_{3} \rightarrow f\left(c_{2}, a_{1}\right), a_{2} \rightarrow f\left(c_{1}, c_{2}\right), a_{1} \rightarrow c_{1}\right\} \\
& R_{2}=\left\{a_{5} \rightarrow g\left(a_{2}, a_{3}\right), \underline{a_{3} \rightarrow a_{2}}, a_{2} \rightarrow d_{1}, a_{1} \rightarrow g\left(c_{1}, c_{1}\right)\right\}
\end{aligned}
$$

In the fourth iteration of the loop, the only non-trivial identity for $a_{4}$ in Step 3 is $a_{4} \approx a_{3}$, which yields the solver $s=a_{3}$ and the new systems:

$$
\begin{aligned}
& R_{1}=\left\{a_{4} \rightarrow f\left(c_{1}, c_{2}\right), a_{3} \rightarrow f\left(c_{2}, a_{1}\right), a_{2} \rightarrow f\left(c_{1}, c_{2}\right), a_{1} \rightarrow c_{1}\right\} \\
& R_{2}=\left\{a_{5} \rightarrow g\left(a_{2}, a_{3}\right), \underline{a_{4} \rightarrow a_{3}}, a_{3} \rightarrow a_{2}, a_{2} \rightarrow d_{1}, a_{1} \rightarrow g\left(c_{1}, c_{1}\right)\right\}
\end{aligned}
$$

Finally, in the fifth iteration of the loop, the only non-trivial identity for $a_{5}$ in Step 3 is $a_{5} \approx a_{4}$, which yields the solver $s=a_{4}$ and the final rewrite systems:

$$
\begin{aligned}
& R_{1}=\left\{\underline{a_{5} \rightarrow a_{4}}, a_{4} \rightarrow f\left(c_{1}, c_{2}\right), a_{3} \rightarrow f\left(c_{2}, a_{1}\right), a_{2} \rightarrow f\left(c_{1}, c_{2}\right), a_{1} \rightarrow c_{1}\right\} \\
& R_{2}=\left\{a_{5} \rightarrow g\left(a_{2}, a_{3}\right), a_{4} \rightarrow a_{3}, a_{3} \rightarrow a_{2}, a_{2} \rightarrow d_{1}, a_{1} \rightarrow g\left(c_{1}, c_{1}\right)\right\}
\end{aligned}
$$

We can now perform the final test on the goal identity $a_{5} \approx a_{4}$. If we test $R_{1} \models_{E_{1}} a_{5} \approx a_{4}$ following the procedure suggested by Lemma 4.3 , we first compute the $R_{1}$-normal forms of $a_{4}, a_{5}$, and then check whether they are equal w.r.t. $E_{1}$. Since both $a_{4}$ and $a_{5}$ rewrite to the same normal form $f\left(c_{1}, c_{2}\right)$, this test clearly succeeds, and thus the procedure answers "yes." Note that the test $R_{2}=E_{E_{2}} a_{5} \approx a_{4}$ succeeds as well, since $a_{4}$ and $a_{5}$ rewrite w.r.t. $R_{2}$ to $d_{1}$ and $g\left(d_{1}, d_{1}\right)$, respectively, and $d_{1} \approx_{E_{2}} g\left(d_{1}, d_{1}\right)$.

\section{Correctness of the combination procedure}

Since the combinations procedure obviously terminates for any input, we only need to prove its soundness and completeness. In the proof below, we will use the notation $R_{1, i}, R_{2, i}$ to denote the updated rewrite systems obtained after step $i$ in the loop $\left(R_{1,0}\right.$ and $R_{2,0}$ are the input systems $R_{1}$ and $\left.R_{2}\right)$.

Proposition 5.1 (Soundness) If the combination procedure answers "yes", then $R_{1} \cup R_{2} \models{ }_{E_{1} \cup E_{2}} u \approx v$.

Proof. Let $i \in\{1, \ldots, n\}$. We start by showing that

$$
R_{1, i} \cup R_{2, i} \models_{E_{1} \cup E_{2}} u \approx v \text { implies } R_{1, i-1} \cup R_{2, i-1} \models_{E_{1} \cup E_{2}} u \approx v \text {. }
$$


First observe that

$$
R_{1, i} \cup R_{2, i}=R_{1, i-1} \cup R_{2, i-1} \cup\left\{a_{i} \approx s\left(\boldsymbol{a}_{i-1}, \boldsymbol{c}, \boldsymbol{d}\right)\right\}
$$

where (i) the term $s\left(\boldsymbol{a}_{i-1}, \boldsymbol{c}, \boldsymbol{d}\right)$ is a local solver of $\varphi\left(\boldsymbol{a}_{i}, \boldsymbol{c}\right)$ w.r.t. the free constant $a_{i}$ in $E_{0}$, and (ii) $\varphi\left(\boldsymbol{a}_{i}, \boldsymbol{c}\right)$ is an $e$-formula such that $R_{j, i-1} \models_{E_{i}} \varphi\left(\boldsymbol{a}_{i}, \boldsymbol{c}\right)$ for some $j \in\{1,2\}$.

Now assume that $R_{1, i} \cup R_{2, i}==_{E_{1} \cup E_{2}} u \approx v$. By (5) above and the fact that the constants $\boldsymbol{d}$ occur only in the solver $s$, we have that

$$
R_{1, i-1} \cup R_{2, i-1} \cup\left\{\exists \boldsymbol{z} \cdot\left(a_{i} \approx s\left(\boldsymbol{a}_{i-1}, \boldsymbol{c}, \boldsymbol{z}\right)\right)\right\} \models_{E_{1} \cup E_{2}} u \approx v .
$$

To prove that $R_{1, i-1} \cup R_{2, i-1} \models_{E_{1} \cup E_{2}} u \approx v$ it is enough to show that $R_{1, i-1} \cup$ $R_{2, i-1} \models_{E_{1} \cup E_{2}} \exists \boldsymbol{z} \cdot\left(a_{i} \approx s\left(\boldsymbol{a}_{i-1}, \boldsymbol{c}, \boldsymbol{z}\right)\right)$. To that end, first observe that $R_{1, i-1} \cup$ $R_{2, i-1} \models_{E_{1} \cup E_{2}} \varphi\left(\boldsymbol{a}_{i}, \boldsymbol{c}\right)$ by monotonicity of $\models$ and (ii) above. Second, by construction of $s$ (see Definition 3.4) and the fact that $E_{1} \cup E_{2}$ extends $E_{0}$ it follows, again by monotonicity, that

$$
R_{1, i-1} \cup R_{2, i-1} \models_{E_{1} \cup E_{2}} \exists \boldsymbol{z} \cdot\left(a_{i} \approx s\left(\boldsymbol{a}_{i-1}, \boldsymbol{c}, \boldsymbol{z}\right)\right) .
$$

Thus, we have completed the proof of Property (4). To prove the proposition now, assume that procedure answers "yes". Then it must be that $R_{1, n} \models{ }_{E_{1}}$ $u \approx v$ which implies that $R_{1, n} \cup R_{2, n} \models_{E_{1} \cup E_{2}} u \approx v$. But then, by a repeated application of Property (4) above, we have that $R_{1} \cup R_{2}=R_{1,0} \cup R_{2,0}={ }_{E_{1} \cup E_{2}}$ $u \approx v$.

The following two lemmas will be useful to prove the completeness of the combination procedure.

Lemma 5.2 Let $\psi_{2}(\boldsymbol{x}, \boldsymbol{y}, \boldsymbol{z})$ be an e-formula in the signature $\Sigma_{0}$ such that $R_{k, i}^{(i)} \models_{E_{k}} \psi_{2}\left(\boldsymbol{a}_{i}, \boldsymbol{c}, \boldsymbol{b}\right)$, where $R_{k, i}^{(i)}, \boldsymbol{a}_{i}$, and $\boldsymbol{c}$ are defined as in the procedure, and $\boldsymbol{b}$ is a set of free constants not in $R_{k, i}^{(i)}$. Then, there is an e-formula $\psi_{0}(\boldsymbol{x}, \boldsymbol{y})$ in the signature $\Sigma_{0}$, such that

$$
R_{k, i}^{(i)} \models E_{k} \psi_{0}\left(\boldsymbol{a}_{i}, \boldsymbol{c}\right) \quad \text { and } \quad \psi_{0}\left(\boldsymbol{a}_{i}, \boldsymbol{c}\right) \models_{E_{0}} \psi_{2}\left(\boldsymbol{a}_{i}, \boldsymbol{c}, \boldsymbol{b}\right) .
$$

Proof. For notational simplicity, we prove the lemma for the case in which $k=1$, as the proof for the case $k=2$ is identical. Let $\Gamma_{0}$ be the set of ground identities $\gamma_{0}$ in the signature $\Sigma_{0}\left(\boldsymbol{a}_{i}, \boldsymbol{c}\right)$ such that $R_{1, i}^{(i)} \models_{E_{1}} \gamma_{0}$ or, equivalently (by treating the rules of $R_{1, i}^{(i)}$ as ground identities), such that $E_{1} \cup R_{1, i}^{(i)} \models \gamma_{0}$. By compactness, it is enough to show that $E_{0} \cup \Gamma_{0} \models \psi_{2}\left(\boldsymbol{a}_{i}, \boldsymbol{c}, \boldsymbol{b}\right)$.

Let $\boldsymbol{c}_{i}$ collect in addition to the elements of $\boldsymbol{c}$ all the other free constants of 
$R_{1, i}^{(i)}$ that do not occur in $\boldsymbol{a}_{i} \cdot{ }^{14}$ Let $F_{1}$ be the $\Sigma_{1}\left(\boldsymbol{a}_{i}, \boldsymbol{c}_{i}\right)$-theory axiomatized by $E_{1} \cup R_{1, i}^{(i)}$ and let $F_{2}$ be the $\Sigma_{0}\left(\boldsymbol{a}_{i}, \boldsymbol{c}, \boldsymbol{b}\right)$-theory axiomatized by $E_{0}$. We construct below two algebras $\mathcal{A}_{1}$ and $\mathcal{A}_{2}$ such that

(1) $\mathcal{A}_{1}$ is a model of $F_{1}$ that $\Sigma_{1}\left(\boldsymbol{a}_{i}, \boldsymbol{c}_{i}\right)$-embeds into a model of $F_{1} \cup E_{0}^{*}$,

(2) $\mathcal{A}_{2}$ is a model of $F_{2}$ that $\Sigma_{0}\left(\boldsymbol{a}_{i}, \boldsymbol{c}, \boldsymbol{b}\right)$-embeds into a model of $F_{2} \cup E_{0}^{*}$ and is initial for $E_{0} \cup \Gamma_{0}$,

(3) $\mathcal{A}_{1}$ and $\mathcal{A}_{2}$ have a common $\Sigma_{0}\left(\boldsymbol{a}_{i}, \boldsymbol{c}\right)$-subalgebra $\mathcal{A}_{0}$.

Given these algebras, by applying Lemma 2.6 to $F_{1}$ and $F_{2}$ we know that there is a model $\mathcal{A}$ of $F_{1} \cup F_{2}$ and embeddings $f_{1}$ and $f_{2}$ of $\mathcal{A}_{1}$ and $\mathcal{A}_{2}$ into $\mathcal{A}$ that agree on $A_{0}$. Since $\mathcal{A}$ is a model of $F_{1} \cup F_{2}$, it is also a $\Sigma_{1}\left(\boldsymbol{a}_{i}, \boldsymbol{c}_{i}, \boldsymbol{b}\right)$-model of $E_{1} \cup R_{1, i}^{(i)}$. Therefore, by the assumption that $R_{1, i}^{(i)} \models_{E_{1}} \psi_{2}\left(\boldsymbol{a}_{i}, \boldsymbol{c}, \boldsymbol{b}\right), \mathcal{A}$ must be a model of (the ground $\Sigma_{0}\left(\boldsymbol{a}_{i}, \boldsymbol{c}, \boldsymbol{b}\right)$-formula) $\psi_{2}\left(\boldsymbol{a}_{i}, \boldsymbol{c}, \boldsymbol{b}\right)$. By Lemma 2.2 and the fact that $\mathcal{A}_{2}$ is $\Sigma_{0}\left(\boldsymbol{a}_{i}, \boldsymbol{c}, \boldsymbol{b}\right)$-embedded into $\mathcal{A}$, we then have that $\mathcal{A}_{2}$ models $\psi_{2}\left(\boldsymbol{a}_{i}, \boldsymbol{c}, \boldsymbol{b}\right)$ as well. Given that $\mathcal{A}_{2}$ is an initial $\Sigma_{0}\left(\boldsymbol{a}_{i}, \boldsymbol{c}_{i}, \boldsymbol{b}\right)$-model of $E_{0} \cup \Gamma_{0}$, it follows that $E_{0} \cup \Gamma_{0} \models \psi_{2}\left(\boldsymbol{a}_{i}, \boldsymbol{c}, \boldsymbol{b}\right)$.

To conclude the proof then we need to define the algebras $\mathcal{A}_{0}, \mathcal{A}_{1}, \mathcal{A}_{2}$ and prove that they satisfy each of the three points above.

For Point 1 , let $\mathcal{C}_{1}$ be the initial $\Sigma_{1}\left(\boldsymbol{c}_{i}\right)$-model of $E_{1}$. Observe that by construction the left-hand side constants of $R_{1, i}^{(i)}$ are exactly $\boldsymbol{a}_{i}$. Therefore, we can use Lemma 4.2 to expand $\mathcal{C}_{1}$ to an initial $\Sigma_{1}\left(\boldsymbol{a}_{i}, \boldsymbol{c}_{i}\right)$-model $\mathcal{A}_{1}$ of $F_{1}=E_{1} \cup R_{1, i}^{(i)}$. To see that $\mathcal{A}_{1}$ embeds into a model of $F_{1} \cup E_{0}^{*}$, first observe that $\mathcal{C}_{1}^{\Sigma_{1}}$ is a finitely generated free model of $E_{1}$ with generators $\boldsymbol{c}_{i}$. By Definition 3.1, there is then a $\Sigma_{1}$-embedding $h$ of $\mathcal{C}_{1}^{\Sigma_{1}}$ into a model of $E_{1} \cup E_{0}^{*}$. Let $\mathcal{B}_{1}$ be the expansion of this model to $\Sigma_{1}\left(\boldsymbol{a}_{i}, \boldsymbol{c}_{i}\right)$ defined by interpreting the constants of $\boldsymbol{a}_{i} \cup \boldsymbol{c}_{i}$ as in $\mathcal{A}_{1}$; that is, by having $d^{\mathcal{B}_{1}}:=h\left(d^{\mathcal{A}_{1}}\right)$ for all $d \in \boldsymbol{a}_{i} \cup \boldsymbol{c}_{i}$. It is not difficult to see that $\mathcal{B}_{1}$ models $F_{1} \cup E_{0}^{*}$ and that $h$ is a $\Sigma_{1}\left(\boldsymbol{a}_{i}, \boldsymbol{c}_{i}\right)$-embedding of $\mathcal{A}_{1}$ into $\mathcal{B}_{1}$.

To prove Point 2, let $\mathcal{A}_{2}$ be an initial $\Sigma_{0}\left(\boldsymbol{a}_{i}, \boldsymbol{c}, \boldsymbol{b}\right)$-model of $E_{0} \cup \Gamma_{0}$. Since $\mathcal{A}_{2}^{\Sigma_{0}}$ is a model of $E_{0}$ and $E_{0}^{*}$ is $E_{0}$ 's model completion, it follows that there is a $\Sigma_{0}$-embedding $h$ of $\mathcal{A}_{2}$ into a $\Sigma_{0}$-model of $E_{0} \cup E_{0}^{*}$. Let $\mathcal{C}_{2}$ be the expansion of this model to $\Sigma_{0}\left(\boldsymbol{a}_{i}, \boldsymbol{c}, \boldsymbol{b}\right)$ defined by interpreting the constants of $\boldsymbol{a}_{i} \cup \boldsymbol{c} \cup \boldsymbol{b}$ as in $\mathcal{A}_{2}$; that is, by having $d^{\mathcal{C}_{2}}:=h\left(d^{\mathcal{A}_{2}}\right)$ for all $d \in \boldsymbol{a}_{i} \cup \boldsymbol{c} \cup \boldsymbol{b}$. It is immediate that $\mathcal{C}_{2}$ models $F_{2} \cup E_{0}^{*}$ and that $h$ is a $\Sigma_{0}\left(\boldsymbol{a}_{i}, \boldsymbol{c}, \boldsymbol{b}\right)$-embedding of $\mathcal{A}_{2}$ into $\mathcal{C}_{2}$.

To prove Point 3, let $\mathcal{A}_{0}$ be an initial $\Sigma_{0}\left(\boldsymbol{a}_{i}, \boldsymbol{c}\right)$-model of $E_{0} \cup \Gamma_{0}$. We first show that $\mathcal{A}_{0}$ can be $\Sigma_{0}\left(\boldsymbol{a}_{i}, \boldsymbol{c}\right)$-embedded into $\mathcal{A}_{1}$. By Lemma 2.1, it is enough to show that $\mathcal{A}_{1}$ satisfies $\Delta_{\emptyset}^{\Sigma_{0}\left(\boldsymbol{a}_{i}, \boldsymbol{c}\right)}\left(\mathcal{A}_{0}\right)$. So let $P$ be a positive ground $\Sigma_{0}\left(\boldsymbol{a}_{i}, \boldsymbol{c}\right)$ literal satisfied by $\mathcal{A}_{0}$. Since $\mathcal{A}_{0}$ is an initial model of $E_{0} \cup \Gamma_{0}$, we have that

$\overline{14}$ These additional constants may arise from the introduction of solvers into $R_{1}$ in previous steps of the procedure. 
$E_{0} \cup \Gamma_{0} \models P$. But then, $E_{1} \cup R_{1, i}^{(i)} \models P$ because $E_{1} \cup R_{1, i}^{(i)} \models E_{0} \cup \Gamma_{0} .{ }^{15}$ Since $\mathcal{A}_{1}$ is a model of $E_{1} \cup R_{1, i}^{(i)}$, we can conclude that $\mathcal{A}_{1}$ satisfies $P$ as well. Now let $\neg P$ be a negative ground $\Sigma_{0}\left(\boldsymbol{a}_{i}, \boldsymbol{c}\right)$-literal satisfied by $\mathcal{A}_{0}$ and assume by contradiction that $\mathcal{A}_{1}$ satisfies $P$. Then, since $\mathcal{A}_{1}$ is an initial model of $E_{1} \cup R_{1, i}^{(i)}$, we have that $E_{1} \cup R_{1, i}^{(i)} \models P$. It follows that $P \in \Gamma_{0}$ and so it must be satisfied by $\mathcal{A}_{0}$, against the assumption that $\mathcal{A}_{0}$ satisfies $\neg P$.

We now show that $\mathcal{A}_{0}$ can be $\Sigma_{0}\left(\boldsymbol{a}_{i}, \boldsymbol{c}\right)$-embedded in $\mathcal{A}_{2}$. First note that, since $\mathcal{A}_{2}$ is an initial $\Sigma_{0}\left(\boldsymbol{a}_{i}, \boldsymbol{c}, \boldsymbol{b}\right)$-model of $E_{0} \cup \Gamma_{0}$, the reduct of $\mathcal{A}_{2}$ to $\Sigma_{0}\left(\boldsymbol{a}_{i}, \boldsymbol{c}\right)$ is a free model of $E_{0} \cup \Gamma_{0}$ over the generators (denoted by) $\boldsymbol{b}$. Since $\mathcal{A}_{0}$ is an initial $\Sigma_{0}\left(\boldsymbol{a}_{i}, \boldsymbol{c}\right)$-model of $E_{0} \cup \Gamma_{0}$, it follows by well-known results on free algebras that $\mathcal{A}_{0}$ can be embedded into that reduct and so can be $\Sigma_{0}\left(\boldsymbol{a}_{i}, \boldsymbol{c}\right)$-embedded into $\mathcal{A}_{2}$.

In conclusion, we have that $\mathcal{A}_{0}$ is $\Sigma_{0}\left(\boldsymbol{a}_{i}, \boldsymbol{c}\right)$-embedded into both $\mathcal{A}_{1}$ and $\mathcal{A}_{2}$. By renaming the elements of $\mathcal{A}_{1}$ and $\mathcal{A}_{2}$ appropriately, we can assume with no loss of generality that these embeddings are in fact inclusions. Hence $\mathcal{A}_{0}$ is a $\Sigma_{0}\left(\boldsymbol{a}_{i}, \boldsymbol{c}\right)$-subalgebra of both $\mathcal{A}_{1}$ and $\mathcal{A}_{2}$, as required by Point 3 .

Lemma 5.3 For every $i=1, \ldots, n$ and every ground e-formula $\psi\left(\boldsymbol{a}_{i}, \boldsymbol{c}\right)$ in the signature $\Sigma_{0}\left(\boldsymbol{a}_{i}, \boldsymbol{c}\right)$,

$$
R_{1, i}^{(i)} \models_{E_{1}} \psi \quad \text { iff } \quad R_{2, i}^{(i)} \models E_{E_{2}} \psi .
$$

In particular, $R_{1, n} \models_{E_{1}} \psi$ iff $R_{2, n} \models_{E_{2}} \psi$ for every ground e-formula $\psi\left(\boldsymbol{a}_{n}, \boldsymbol{c}\right)$ in the signature $\Sigma_{0}\left(\boldsymbol{a}_{n}, \boldsymbol{c}\right)$.

Proof. We prove the lemma by induction on $i$. The base case $i=0$ is trivial since $R_{1,0}^{(0)}$ and $R_{2,0}^{(0)}$ are empty, and $E_{1}, E_{2}$ are conservative extensions of the same theory $E_{0}$ over $\Sigma_{0}$.

Thus, let $i>0$ and assume that the lemma holds for $i-1$. Let $j, k, t_{i}, \varphi\left(\boldsymbol{a}_{i}, \boldsymbol{c}\right)$, and $s\left(\boldsymbol{a}_{i-1}, \boldsymbol{c}, \boldsymbol{d}\right)$ be defined as in the $i$-th iteration of the loop in the combination procedure. Then we have $R_{j, i}=R_{j, i-1}$ and $R_{k, i}=R_{k, i-1} \cup\left\{a_{i} \rightarrow\right.$ $\left.s\left(\boldsymbol{a}_{i-1}, \boldsymbol{c}, \boldsymbol{d}\right)\right\}$.

First, we show that $R_{j, i}^{(i)} \models_{E_{j}} \psi$ implies $R_{k, i}^{(i)} \models_{E_{k}} \psi$. Observe that $R_{j, i}^{(i)}$ is equal to $R_{j, i-1}^{(i)}$ and that $R_{k, i}^{(i)}$ is equal to to $R_{k, i-1}^{(i-1)} \cup\left\{a_{i} \rightarrow s\left(\boldsymbol{a}_{i-1}, \boldsymbol{c}, \boldsymbol{d}\right)\right\}$. From $R_{j, i}^{(i)} \models_{E_{j}} \psi$ it follows that $\varphi \models_{E_{0}} \psi$ (since, modulo $E_{0}$, every conjunct of $\psi$ occurs as a conjunct in $\varphi$ by the definition of $\varphi$ ). Thus, it is sufficient to show that $R_{k, i}^{(i)} \models_{E_{k}} \varphi$. Because $a_{i} \rightarrow s\left(\boldsymbol{a}_{i-1}, \boldsymbol{c}, \boldsymbol{d}\right)$ belongs to $R_{k, i}^{(i)}$ and since $s\left(\boldsymbol{a}_{i-1}, \boldsymbol{c}, \boldsymbol{d}\right)$ is a local solver of $\varphi$ w.r.t. $a_{i}$, it is sufficient to show that the corresponding solvability condition $C\left(\boldsymbol{a}_{i-1}, \boldsymbol{c}\right)$ follows from $E_{k}$ and $R_{k, i}^{(i)}$. However,

$\overline{{ }^{15} E_{1} \cup R_{1, i}^{(i)}} \models \Gamma_{0}$ by construction of $\Gamma_{0}$, and $E_{1} \cup R_{1, i}^{(i)} \models E_{0}$ because $E_{1}$ extends $E_{0}$. 
this formula does not contain $a_{i}$, and thus we can argue as follows. Since $\varphi$ implies its own solvability condition (in $E_{0}$, and thus also in $E_{j}$ ), $R_{j, i}^{(i)} \models_{E_{j}} \varphi$ implies $R_{j, i}^{(i)}=E_{E_{j}} C\left(\boldsymbol{a}_{i-1}, \boldsymbol{c}\right)$. Because $C\left(\boldsymbol{a}_{i-1}, \boldsymbol{c}\right)$ does not contain $a_{i}$ and since $R_{j, i}=R_{j, i-1}$, this implies that $R_{j, i-1}^{(i-1)} \models_{E_{j}} C\left(\boldsymbol{a}_{i-1}, \boldsymbol{c}\right)$ by Lemma 4.3. ${ }^{16}$ Thus, the induction hypothesis yields $R_{k, i-1}^{(i-1)} \models_{E_{k}} C\left(\boldsymbol{a}_{i-1}, \boldsymbol{c}\right)$. Since $R_{k, i-1}^{(i-1)} \subseteq R_{k, i}^{(i)}$, this finally implies $R_{k, i}^{(i)} \models_{E_{k}} C\left(\boldsymbol{a}_{i-1}, \boldsymbol{c}\right)$. In conclusion, we have shown that $R_{k, i}^{(i)}={ }_{E_{k}} \psi$.

Second, we show that $R_{k, i}^{(i)} \models_{E_{k}} \psi$ implies $R_{j, i}^{(i)} \models_{E_{j}} \psi$. Since $R_{k, i}:=R_{k, i-1} \cup$ $\left\{a_{i} \rightarrow s\left(\boldsymbol{a}_{i-1}, \boldsymbol{c}, \boldsymbol{d}\right)\right\}$, we know (again by Lemma 4.3) that $R_{k, i}^{(i)} \models_{E_{k}} \psi$ implies that $R_{k, i-1}^{(i-1)} \models_{E_{k}} \psi_{2}\left(\boldsymbol{a}_{i-1}, \boldsymbol{c}, \boldsymbol{d}\right)$ where $\psi_{2}$ is obtained from $\psi$ by replacing every occurrence of $a_{i}$ by $s\left(\boldsymbol{a}_{i-1}, \boldsymbol{c}, \boldsymbol{d}\right)$. Applying Lemma 5.2 to $R_{k, i-1}^{(i-1)} \models{ }_{E_{k}}$ $\psi_{2}\left(\boldsymbol{a}_{i-1}, \boldsymbol{c}, \boldsymbol{d}\right)$, we then obtain an $e$-formula $\psi_{0}\left(x_{1}, \ldots, x_{i-1}, \boldsymbol{y}\right)$ in the shared signature $\Sigma_{0}$ such that

(1) $R_{k, i-1}^{(i-1)}==_{E_{k}} \psi_{0}\left(\boldsymbol{a}_{i-1}, \boldsymbol{c}\right)$ and

(2) $\psi_{0}\left(\boldsymbol{a}_{i-1}, \boldsymbol{c}\right) \models_{E_{0}} \psi_{2}\left(\boldsymbol{a}_{i-1}, \boldsymbol{c}, \boldsymbol{d}\right)$.

By applying the induction hypothesis to the first entailment, we then have $R_{j, i-1}^{(i-1)} \models_{E_{j}} \psi_{0}\left(\boldsymbol{a}_{i-1}, \boldsymbol{c}\right)$, and so, since $R_{j, i-1}=R_{j, i}$, also $R_{j, i}^{(i)} \models_{E_{j}} \psi_{0}\left(\boldsymbol{a}_{i-1}, \boldsymbol{c}\right)$.

By the substitutivity property of equality and the construction of $\psi_{2}$, the second entailment implies that $\psi_{0}\left(\boldsymbol{a}_{i-1}, \boldsymbol{c}\right) \wedge a_{i} \approx s\left(\boldsymbol{a}_{i-1}, \boldsymbol{c}, \boldsymbol{d}\right) \models_{E_{0}} \psi$, which is equivalent to

$$
\psi_{0}\left(\boldsymbol{a}_{i-1}, \boldsymbol{c}\right) \wedge \exists \boldsymbol{z} .\left(a_{i} \approx s\left(\boldsymbol{a}_{i-1}, \boldsymbol{c}, \boldsymbol{z}\right)\right) \models_{E_{0}} \psi
$$

as the constants $\boldsymbol{d}$ do not occur in $\psi$. Given that $s\left(\boldsymbol{a}_{i-1}, \boldsymbol{c}, \boldsymbol{z}\right)$ is a local solver for $\varphi\left(\boldsymbol{a}_{i}, \boldsymbol{c}\right)$, we have by Definition 3.4 that $\varphi\left(\boldsymbol{a}_{i}, \boldsymbol{c}\right) \models_{E_{0}} \exists \boldsymbol{z} .\left(a_{i} \approx s\left(\boldsymbol{a}_{i-1}, \boldsymbol{c}, \boldsymbol{z}\right)\right)$. It follows that $\left\{\psi_{0}, \varphi\right\} \models{ }_{E_{0}} \psi$.

Recalling that $R_{j, i}^{(i)} \models E_{j} \varphi$ by construction of $\varphi$ and that $R_{j, i}^{(i)} \models_{E_{j}} \psi_{0}$ as shown above, we can conclude that $R_{j, i}^{(i)} \models{ }_{E_{j}} \psi$.

Proposition 5.4 (Completeness) If $R_{1} \cup R_{2} \models_{E_{1} \cup E_{2}} u \approx v$, then the combination procedure answers "yes".

Proof. Since the procedure is terminating, it is enough to show that $R_{1,0} \cup$ $R_{2,0} \forall E_{E_{1} \cup E_{2}} u \approx v$ whenever the combination procedure answer "no". We do that by building a model of $R_{1,0} \cup R_{2,0} \cup E_{1} \cup E_{2}$ that falsifies $u \approx v$.

$\overline{{ }^{16} \text { Lemma }} 4.3$ applies here because $C\left(\boldsymbol{a}_{i-1}, \boldsymbol{c}\right)$ is a conjunction of identities, and so it is entailed by a set of formulae iff each of its identities is. 
Assume then that the combination procedure answer "no" and let $k \in\{1,2\}$. Where $\boldsymbol{c}$ is defined as in Figure 1 and $\boldsymbol{d}_{k}$ is a tuple collecting all the new constants introduced in the rewrite system $R_{k}$ during execution of the procedure (see Step 4 of the loop), let $\mathcal{A}_{k, 0}$ be the initial model of $E_{k}$ over the signature $\Sigma_{k}\left(\boldsymbol{c}, \boldsymbol{d}_{k}\right)$. By Lemma $4.2, \mathcal{A}_{k, 0}$ can be expanded to a $\Sigma_{k}\left(\boldsymbol{a}_{n}, \boldsymbol{c}, \boldsymbol{d}_{k}\right)$-algebra $\mathcal{A}_{k}$, which is the initial $\Sigma_{k}\left(\boldsymbol{a}_{n}, \boldsymbol{c}, \boldsymbol{d}_{k}\right)$-model of the theory $E_{k} \cup R_{k, n}$.

In particular, the fact that $\mathcal{A}_{k}$ is initial implies for every ground $e$-formula $\varphi$ in the signature $\Sigma_{0}\left(\boldsymbol{a}_{n}, \boldsymbol{c}\right)$,

$$
\mathcal{A}_{k} \text { satisfies } \varphi \text { iff } \quad R_{k, n} \models_{E_{k}} \varphi \text {. }
$$

Now, let $\mathcal{B}_{k}$ be the $\Sigma_{0}$-subalgebra of $\mathcal{A}_{k}$ generated by (the interpretations in $\mathcal{A}_{k}$ of) the constants $\boldsymbol{a}_{n} \cup \boldsymbol{c}$. We claim that the algebras $\mathcal{B}_{1}$ and $\mathcal{B}_{2}$ satisfy each other's diagram. To see that, let $\psi$ be a ground $\Sigma_{0}\left(\boldsymbol{a}_{n}, \boldsymbol{c}\right)$-identity. Then,

$$
\begin{aligned}
\psi \in \Delta_{\boldsymbol{a}_{n} \cup \boldsymbol{c}}^{\Sigma_{0}}\left(\mathcal{B}_{k}\right) & \text { iff } \left.\mathcal{B}_{k} \text { satisfies } \psi \text { [by definition of } \Delta_{\boldsymbol{a}_{n} \cup \boldsymbol{c}}^{\Sigma_{0}}\left(\mathcal{B}_{k}\right)\right] \\
& \text { iff } \mathcal{A}_{k} \text { satisfies } \psi \text { [by construction of } \mathcal{B}_{k} \text { and Lemma 2.2] } \\
& \text { iff } R_{k, n} \models_{E_{k}} \psi \quad[\text { by (6) above]. }
\end{aligned}
$$

By Lemma 5.3, we can conclude that $\psi \in \Delta_{a \cup \boldsymbol{c}}^{\Sigma_{0}}\left(\mathcal{B}_{1}\right)$ iff $\psi \in \Delta_{a \cup \boldsymbol{c}}^{\Sigma_{0}}\left(\mathcal{B}_{2}\right)$. It follows from the observation after Lemma 2.1 that $\mathcal{B}_{1}$ and $\mathcal{B}_{2}$ are $\Sigma_{0}$-isomorphic, hence they can be identified with no loss of generality. Therefore, let $\mathcal{A}_{0}:=\mathcal{B}_{1}=\mathcal{B}_{2}$ and observe that for $k=1,2$,

(1) $\mathcal{A}_{k}^{\Sigma_{k}}$ is, by construction, a finitely generated free model of $E_{k},{ }^{17}$ and so by the $E_{0}$-compatibility of $E_{k}$ it embeds into a model of $E_{k} \cup E_{0}^{*}$;

(2) $\mathcal{A}_{0}$ is a $\Sigma_{0}$-subalgebra of $\mathcal{A}_{k}^{\Sigma_{k}}$;

(3) $\mathcal{A}_{0}$ is a model of $E_{0}$-because $\mathcal{A}_{k}^{\Sigma_{0}}$ is a model of $E_{0}$ and the set of models of an equational theory is closed under building subalgebras.

By Lemma 2.6 it follows that there is a model $\mathcal{A}$ of $E_{1} \cup E_{2}$ such that for $k=1,2$ there is a $\Sigma_{k}$-embedding $f_{k}$ of $\mathcal{A}_{k}^{\Sigma_{k}}$ into $\mathcal{A}$. By the same lemma we also have that $f_{1}\left(c^{\mathcal{A}_{1}}\right)=f_{2}\left(c^{\mathcal{A}_{2}}\right)$ for all $c \in \boldsymbol{a}_{n} \cup \boldsymbol{c}$, the generators of $\mathcal{A}_{0}$. Let then $\mathcal{A}^{\prime}$ be the expansion of $\mathcal{A}$ to the signature $\left(\Sigma_{1} \cup \Sigma_{2}\right)\left(\boldsymbol{a}_{n}, \boldsymbol{c}\right)$ such that $c^{\mathcal{A}^{\prime}}=f_{1}\left(c^{\mathcal{A}_{1}}\right)$ for every $c \in \boldsymbol{a}_{n} \cup \boldsymbol{c}$. It is not difficult to see that $f_{k}$ is a $\Sigma_{k}\left(\boldsymbol{a}_{n}, \boldsymbol{c}\right)$-embedding of $\mathcal{A}_{k}$ into $\mathcal{A}^{\prime}$ for $k=1,2$.

Observe that $\mathcal{A}^{\prime}$, which is clearly a model of $E_{1} \cup E_{2}$, is also a model of $R_{1,0} \cup R_{2,0}$. In fact, by construction of $R_{1, n}$ and $R_{2, n}$, for all $a \rightarrow t \in R_{1,0} \cup R_{2,0}$, there is a $k \in\{1,2\}$ such that $a \rightarrow t \in R_{k, n}$. It follows immediately that $R_{k, n} \models_{E_{k}} a \approx t$, which implies by (6) above that $\mathcal{A}_{k}$ satisfies $a \approx t$. But then $\mathcal{A}^{\prime}$ satisfies $a \approx t$ as well by Lemma 2.2 .

\footnotetext{
$\overline{{ }^{17} \text { With }}$ generators $\boldsymbol{c} \cup \boldsymbol{d}_{k}$.
} 
In conclusion, we have that $\mathcal{A}^{\prime}$ is a $\left(\Sigma_{1} \cup \Sigma_{2}\right)\left(\boldsymbol{a}_{n}, \boldsymbol{c}\right)$-model of $R_{1,0} \cup R_{2,0} \cup E_{1} \cup E_{2}$. All we need to show then is that $\mathcal{A}^{\prime}$ falsifies $u \approx v$. Now, since the procedure returns "no" by assumption, it must be that $R_{1, n} \forall_{E_{1}} u \approx v$. We then have that $\mathcal{A}_{1}$ falsifies $u \approx v$ by (6) above and $\mathcal{A}^{\prime}$ falsifies $u \approx v$ by Lemma 2.2.

Note that in the last paragraph of the proof above we could have given a completely symmetrical argument if the final test in the procedure had been on whether $R_{2, n} \models_{E_{2}} u \approx v$. In other words, the procedure's completeness does not depend on which component theory is used for the final test.

From the total correctness of the combination procedure, we then obtain the following modular decidability result.

Theorem 5.5 Let $E_{0}, E_{1}, E_{2}$ be three equational theories of respective signature $\Sigma_{0}, \Sigma_{1}, \Sigma_{2}$ such that

- $\Sigma_{0}=\Sigma_{1} \cap \Sigma_{2}$;

- $E_{0}$ is Gaussian and effectively locally finite;

- for $i=1,2, E_{i}$ is $E_{0}$-compatible and a conservative extension of $E_{0}$.

If the word problem in $E_{1}$ and in $E_{2}$ is decidable, then the word problem in $E_{1} \cup E_{2}$ is also decidable.

An immediate consequence of the above result is that, if the shared theory $E_{0}$ of $E_{1}$ and $E_{2}$ is absolutely combinable, then the word problem is decidable in $E_{1} \cup E_{2}$ iff it is decidable in $E_{1}$ and $E_{2}$ separately. In fact, the conditions of Theorem 5.5 are all satisfied, with the possible exception that one of the two theories may not be a conservative extension of $E_{0}$. However, if this is the case, say for $E_{1}$, then $E_{1}$ is trivial (because $E_{0}$ is absolutely combinable). Hence $E_{1} \cup E_{2}$ is trivial as well, and thus the word problem in $E_{1} \cup E_{2}$ is trivially decidable.

In particular, from the absolute combinability of the pure equality theory $E_{=}$ (see Example 3.7), we obtain as a corollary to Theorem 5.5 the well-known decidability result for the word problem in the union of two equational theories with disjoint signatures and decidable word problems (see, e.g., [3]).

Example 3.6 concerning the absolute combinability of the theory $T_{K}$ of vector spaces over a finite field $K$ shows further applications of Theorem 5.5: for instance, one can take as $E_{1}$ the theory of $K$-algebras, ${ }^{18}$ as $E_{2}$ the theory of vector spaces with an endomorphism, and obtain as a consequence the decidability of the word problem for the theory $E_{1} \cup E_{2}$ of $K$-algebras over a finite field $K$ endowed with a linear endomorphism.

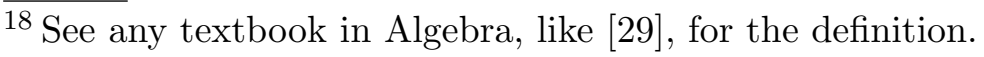


In the next section, we use Theorem 5.5 to show that the decidability of validity transfers from classical modal logics to their fusion.

\section{Fusion decidability in modal logics}

In this section, we first show that the theory of Boolean algebras is absolutely combinable. This allows us then to apply Theorem 5.5 to show a strong transfer result for decidability of validity from modal logics to their fusion. The modal logics to which this result applies are called classical modal logics in the literature.

\subsection{Boolean algebras}

The theory $B A$ of Boolean algebras is the equational theory over the signature $\{\cap, \cup, \overline{(-)}, 1,0\}$ given by the following identities:

$$
\begin{aligned}
x \cap y & \approx y \cap x \\
x \cap(y \cap z) & \approx(x \cap y) \cap z \\
(x \cap y) \cup y & \approx y \\
x \cap(y \cup z) & \approx(x \cap y) \cup(x \cap z) \\
x \cap x & \approx x \\
x \cap 0 & \approx 0 \\
x \cap 1 & \approx x \\
x \cap \bar{x} & \approx 0
\end{aligned}
$$

$$
\begin{aligned}
x \cup y & \approx y \cup x \\
x \cup(y \cup z) & \approx(x \cup y) \cup z \\
(x \cup y) \cap y & \approx y \\
x \cup(y \cap z) & \approx(x \cup y) \cap(x \cup z) \\
x \cup x & \approx x \\
x \cup 0 & \approx x \\
x \cup 1 & \approx 1 \\
x \cup \bar{x} & \approx 1
\end{aligned}
$$

It is well-known that $B A$ is locally finite. In fact, let $\boldsymbol{c}=\left(c_{1}, \ldots, c_{n}\right)$ be a finite collection of free constants. Every Boolean ground term over the constants in $\boldsymbol{c}$ is equivalent in $B A$ to a term in "conjunctive normal form," a meet of terms of the form $d_{1} \cup \cdots \cup d_{n}$, where each $d_{i}$ is either $c_{i}$ or $\bar{c}_{i}$. It is easy to see that the set $R_{B A}(\boldsymbol{c})$ of such normal forms is isomorphic to the powerset of the powerset of $\boldsymbol{c}$, which is effectively computable and has cardinality $2^{2^{n}}$. Hence we have the following result:

Proposition 6.1 BA is effectively locally finite.

It is not possible to extend $B A$ with proper axioms in its own signature: in fact, as soon as one extends $B A$ with an axiom $s \approx t$ for any $s$ and $t$ such that $s \not_{B A} t$, the equation $0 \approx 1$ becomes valid. This can be shown by an appropriate instantiation of the variables of $s \approx t$ by 0 and 1 , followed by simple Boolean simplifications. The validity of $0 \approx 1$ in turn makes all Boolean 
terms equivalent to 0 (as one can easily show), making the extension a trivial equational theory. Thus we have the following result:

Proposition 6.2 All non-trivial equational extensions of $B A$ are conservative extensions of $B A$.

Recall (e.g., from $[27,30]$ ) that $B A$ admits as a model completion the theory of atomless Boolean algebras. ${ }^{19}$ A Boolean algebra $\mathcal{B}$ is said to be atomless iff it does not have atoms, where an atom is a nonzero element $a \in \mathcal{B}$ such that for all $b \in \mathcal{B}$ either $a \leq b$ or $a \leq \bar{b}{ }^{20}$

Proposition 6.3 If $E$ is a non-trivial equational extension of $B A$, then $E$ is BA-compatible.

Proof. We need to embed any finitely generated $E$-free algebra into a model of $E$ whose Boolean reduct is atomless. Since any finitely generated $E$-free algebra can be embedded into the $E$-free algebra $\mathcal{A}$ with a countably infinite set $X$ of generators, it is sufficient to show that this algebra $\mathcal{A}$ is atomless.

We know that $\mathcal{A}=T(\Sigma, X) / \approx_{E}$ where $\Sigma$ is the signature of $E$. Take a candidate atom $a=[t]_{\approx_{E}}$ for some term $t \in T(\Sigma, X)$. Pick a variable $x \in X$ that does not occur in $t$ (this is possible as $X$ is infinite). For $[t]_{\approx_{E}}$ to be an atom we must have in $\mathcal{A}$ either $[t]_{\approx_{E}} \leq[x]_{\approx_{E}}$ or $[t]_{\approx_{E}} \leq[\bar{x}]_{\approx_{E}}$, but in both cases this yields $[t]_{\approx_{E}}=0$. In fact, in the former case, we have

$$
\models_{E} t \cap x \approx t,
$$

so that if we replace $x$ by 0 , we get $\models_{E} 0 \approx t$, proving that in fact $a=[t]_{\approx_{E}}=0$ is not an atom. The latter case is analogous: we can just use 1 instead of 0 in the argument above.

To prove that $B A$ is absolutely combinable, it remains to be shown that it is Gaussian. This is done in the next subsection.

\subsection{Boolean solved forms}

Since we will make essential use of results from the Boolean unification literature, we prefer to switch temporarily to a Boolean ring notation, commonly adopted in that literature. It should be recalled anyway that Boolean algebras

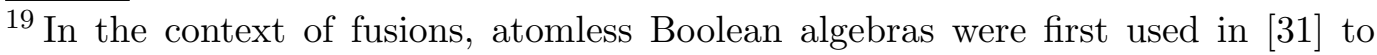
prove that fusions are conservative extensions of their components. The proof of the decidability transfer result in [15] also makes use of atomless Boolean algebras.

${ }^{20}$ Where $a \leq b$ means that $a \cap b=a$.
} 
and Boolean rings are essentially the same theory, expressed in different signatures. The difference is merely a notational question: one can convert terms in the signature of Boolean algebras into terms in the signature of Boolean rings and vice versa, the conversion being bijective modulo the axioms of the respective theories. The theory $B R$ of Boolean rings is the theory in the signature $\Sigma_{B R}=\{+, *, 0,1\}$, one of whose possible axiomatizations is the following:

$$
\begin{aligned}
x * y & \approx y * x, & x+y & \approx y+x, \\
x *(y * z) & \approx(x * y) * z, & x+(y+z) & \approx(x+y)+z, \\
x *(y+z) & \approx(x * y)+(x * z), & x * x & \approx x, \\
x+x & \approx 0, & x * 0 & \approx 0, \\
x+0 & \approx x, & x * 1 & \approx x .
\end{aligned}
$$

It is well-known that when working with $e$-formulae in the theory $B A$, it is enough to consider only $e$-formulae of the form $t \approx 1$. The reason is that for every $e$-formula $\varphi$ of the form $s_{1} \approx t_{1} \wedge \cdots \wedge s_{n} \approx t_{n}$ in the signature of $B A$ the following first-order equivalence holds: ${ }^{21}$

$$
\models_{B A} \varphi \Leftrightarrow\left(\left(s_{1} \supset t_{1}\right) \cap\left(t_{1} \supset s_{1}\right) \cap \cdots \cap\left(s_{n} \supset t_{n}\right) \cap\left(t_{n} \supset s_{n}\right)\right) \approx 1
$$

Note that the symbol $\Leftrightarrow$ here denotes bi-implication at the first order logic level; it should not be confused with bi-implication at the level of modal logics or of Boolean algebra terms.

In a similar way, when working with $e$-formulae in the theory $B R$, it is enough to consider only $e$-formulae of the form $t \approx 0$. The reason now is that, for every $e$-formula $\varphi$ of the form $s_{1} \approx t_{1} \wedge \cdots \wedge s_{n} \approx t_{n}$ in the signature of $B R$ the following equivalence holds:

$$
\models_{B R} \varphi \Leftrightarrow\left(\left(\left(s_{1}+t_{1}+1\right) * \cdots *\left(s_{n}+t_{n}+1\right)\right)+1\right) \approx 0 .
$$

We show below that every formula of the form $t(\boldsymbol{x}, y) \approx 0$ can be effectively turned into the conjunction of a solvability condition $c(\boldsymbol{x}) \approx 0$ and of a local solver parametrization $\exists z \cdot(y \approx s(\boldsymbol{x}, z))$. It then follows immediately by Definition 3.4 that $B R$ is Gaussian. As a consequence, $B A$ is Gaussian as well.

We will use the following general result, adapted from [21], on the computation of most general BR-unifiers based on Löwnheim's formula.

Proposition 6.4 Let $t(\boldsymbol{c}, y) \approx 0$ be a BR-unification problem with (free) constants $\boldsymbol{c}$ and (only) variable $y$. For all unifiers $\{y \mapsto r(\boldsymbol{c})\}$ of $t(\boldsymbol{c}, y) \approx 0$ and

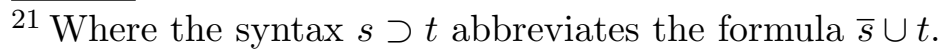


fresh variables $z$, the substitution

$$
\{y \mapsto z+t(\boldsymbol{c}, z) *(z+r(\boldsymbol{c}))\}
$$

is a most general BR-unifier of $t(\boldsymbol{c}, y) \approx 0$.

We will also need the next two lemmas.

Lemma 6.5 Let $t(\boldsymbol{x}, y)$ be any $\Sigma_{B R}$-term and let $c(\boldsymbol{x})=t(\boldsymbol{x}, 1) * t(\boldsymbol{x}, 0)$. Then,

$$
\models_{B R} \quad c(\boldsymbol{x}) *(1+t(\boldsymbol{x}, y)) \approx 0
$$

Proof. To prove the claim we can use the fact that the two-element Boolean ring $\mathcal{B}_{2}$, with carrier $\{0,1\}$, generates the whole variety of Boolean rings. ${ }^{22}$ Then, it is enough to check that $c(\boldsymbol{x}) *(1+t(\boldsymbol{x}, y))$ evaluates to 0 for every assignment $V$ of the variables $y, \boldsymbol{x}$ into $\{0,1\}$.

Let $V$ be such an assignment and for every term $u$ let $V[u]$ be the value denoted by $u$ in $\mathcal{B}_{2}$ under the assignment $V$. If $V[t(\boldsymbol{x}, y)]=1$, the claim follows immediately from the axioms of $B R$. If instead $V[t(\boldsymbol{x}, y)]=0$, depending on whether $V[y]=1$ or $V[y]=0$, we have also $V[t(\boldsymbol{x}, 1)]=0$ or $V[t(\boldsymbol{x}, 0)]=0$ and in any case $V[c(\boldsymbol{x})]=0$.

The next lemma is related to Boole's method for computing most general $B R$-unifiers [21].

Lemma 6.6 Let $t(\boldsymbol{x}, y)$ be a $\Sigma_{B R}$-term and let $c(\boldsymbol{x})=t(\boldsymbol{x}, 1) * t(\boldsymbol{x}, 0)$. The substitution $\sigma:=\{y \mapsto 1+t(\boldsymbol{x}, 1)\}$ is a BR-unifier of the unification problem

$$
t(\boldsymbol{x}, y) *(1+c(\boldsymbol{x})) \approx 0
$$

in which the elements of $\boldsymbol{x}$ are treated as (free) constants and $y$ is the only variable.

Proof. For notational convenience, let us denote the term $t \sigma$ obtained by applying the substitution $\sigma$ to $t$ by $t(\boldsymbol{x}, 1+t(\boldsymbol{x}, 1))$. Let $\mathcal{B}_{2}$ be again the two-element Boolean ring with carrier $\{0,1\}$ as in the proof of Lemma 6.5. It is enough to show that the term

$$
u=t(\boldsymbol{x}, 1+t(\boldsymbol{x}, 1)) *(1+c(\boldsymbol{x}))
$$

evaluates to 0 for every assignment of the variables $y, \boldsymbol{x}$ into $\{0,1\}$.

$\overline{{ }^{22} \text { This }}$ means that an identity is entailed by $B R$ iff it is valid in $\mathcal{B}_{2}$. This may be seen as a consequence e.g. of Stone representation theorem [32], saying that any Boolean ring embeds into a Cartesian power of $\mathcal{B}_{2}$. 
Let $V$ be such an assignment. If $V[c(\boldsymbol{x})]=1$, the whole term $u$ trivially evaluates to 0 . Therefore, suppose that $V[c(\boldsymbol{x})]=0$. Then it is enough to show that $V[t(\boldsymbol{x}, 1+t(\boldsymbol{x}, 1))]=0$. Since $V[c(\boldsymbol{x})]=0$, from the definition of $c(\boldsymbol{x})$, it must be that either (i) $V[t(\boldsymbol{x}, 1)]=0$ or (ii) $V[t(\boldsymbol{x}, 1)]=1$ and $V[t(\boldsymbol{x}, 0)]=0$. In the first case, we get that $V[t(\boldsymbol{x}, 1+t(\boldsymbol{x}, 1))]=V[t(\boldsymbol{x}, 1)]=0$. In the second case, we get that $V[t(\boldsymbol{x}, 1+t(\boldsymbol{x}, 1))]=V[t(\boldsymbol{x}, 0)]=0$.

We are now ready to prove the existence (and computability) of solvability conditions and local solvers in $B R$ for all $e$-formulae of the form $t(\boldsymbol{x}, y) \approx 0$.

Proposition 6.7 For every $\Sigma_{B R}$-term $t(\boldsymbol{x}, y)$, there exist $\Sigma_{B R}$-terms $c(\boldsymbol{x})$ and $s(\boldsymbol{x}, z)$, computable from $t$ in linear time, such that

$$
\models_{B R} t(\boldsymbol{x}, y) \approx 0 \Leftrightarrow(c(\boldsymbol{x}) \approx 0 \wedge \exists z .(y \approx s(\boldsymbol{x}, z)))
$$

Proof. Let

$$
c(\boldsymbol{x})=t(\boldsymbol{x}, 1) * t(\boldsymbol{x}, 0)
$$

as in Lemmas 6.5 and 6.6. We show that we can define a local solver $s(\boldsymbol{x}, z)$ for $t(\boldsymbol{x}, y) \approx 0$ based on the solvability condition $c(\boldsymbol{x}) \approx 0$.

By Lemma 6.6, the substitution $\{y \mapsto 1+t(\boldsymbol{x}, 1)\}$ is a $B R$-unifier of the unification problem

$$
t(\boldsymbol{x}, y) *(1+c(\boldsymbol{x})) \approx 0 .
$$

By Proposition 6.4 then, where $z$ is a fresh variable and

$$
s(\boldsymbol{x}, z):=z+t(\boldsymbol{x}, z) *(1+c(\boldsymbol{x})) *(z+1+t(\boldsymbol{x}, 1))
$$

the substitution $\{y \mapsto s(\boldsymbol{x}, z)\}$ is a most general $B R$-unifier of (8), which means in particular that $s(\boldsymbol{x}, z)$ is a solution of (8), i.e.,

$$
\models_{B R} t(\boldsymbol{x}, s(\boldsymbol{x}, z)) *(1+c(\boldsymbol{x})) \approx 0 .
$$

We use (10) to show that

(i) $t(\boldsymbol{x}, y) \approx 0 \quad=_{B R} c(\boldsymbol{x}) \approx 0 \wedge \exists z \cdot(y \approx s(\boldsymbol{x}, z))$ and

(ii) $\left.c(\boldsymbol{x}) \approx 0 \wedge \exists z \cdot(y \approx s(\boldsymbol{x}, z))\right|_{B R} t(\boldsymbol{x}, y) \approx 0$,

from which the proposition's equivalence immediately follows.

(i) Let $\mathcal{B}$ be any model of $B R$ and let $V$ be any assignment of the variables $\boldsymbol{x}, y$ into $\mathcal{B}$ such that $V[t(\boldsymbol{x}, y)]=0 .{ }^{23}$ Then extend $V$ to $z$ by letting $V[z]=V[y]$. From Lemma 6.5 (and the axioms of $B R$ ) we can deduce that $V[c(\boldsymbol{x})]=0$ and

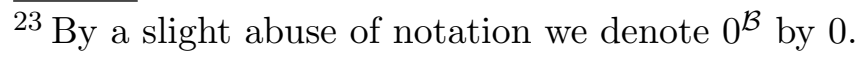




$$
\begin{aligned}
V[s(\boldsymbol{x}, z)] & =V[s(\boldsymbol{x}, y)] \\
& =V[y+t(\boldsymbol{x}, y) *(1+c(\boldsymbol{x})) *(y+1+t(\boldsymbol{x}, 1))] \\
& =V[y+0 *(1+c(\boldsymbol{x})) *(y+1+t(\boldsymbol{x}, 1))] \\
& =V[y+0]=V[y] .
\end{aligned}
$$

It follows that $\mathcal{B}$ satisfies $c(\boldsymbol{x}) \approx 0 \wedge \exists z .(y \approx s(\boldsymbol{x}, z))$ under the assignment $V$, which proves claim (i).

(ii) Let $\mathcal{B}$ be any model of $B R$ and let $V$ be any assignment of $\boldsymbol{x}, y$ into $\mathcal{B}$ such that $\mathcal{B}$ satisfies $c(\boldsymbol{x}) \approx 0 \wedge \exists z .(y \approx s(\boldsymbol{x}, z))$. Clearly, it is possible to extend $V$ to $z$ so that $V[c(\boldsymbol{x})]=0$ and $V[y]=V[s(\boldsymbol{x}, z)]$. Together with (10), we then have

$$
\begin{aligned}
V[t(\boldsymbol{x}, y)] & =V[t(\boldsymbol{x}, s(\boldsymbol{x}, z))] \\
& =V[t(\boldsymbol{x}, s(\boldsymbol{x}, z)) *(1+0)] \\
& =V[t(\boldsymbol{x}, s(\boldsymbol{x}, z)) *(1+c(\boldsymbol{x}))]=0 .
\end{aligned}
$$

It follows that $\mathcal{B}$ satisfies $t(\boldsymbol{x}, y) \approx 0$ under the assignment $V$, which proves claim (ii).

To conclude the proof, we need to show that $c(\boldsymbol{x})$ and $s(\boldsymbol{x}, y)$ are computable in linear time from $t(\boldsymbol{x}, y) \approx 0$. This, however, is immediate from the explicit definitions we have provided for them above.

Strictly speaking, the result above proves that the theory $B R$ of Boolean rings, not the theory $B A$ of Boolean algebras, is Gaussian. However, given an $e$-formula $u(\boldsymbol{x}, y) \approx 1$ in the signature $\Sigma_{B A}$, one can translate it into a corresponding formula $t(\boldsymbol{x}, y) \approx 0$, compute a satisfiability condition and local solver for $t(\boldsymbol{x}, y) \approx 0$ in $B R$, and translate those back into a satisfiability condition and local solver for $u(\boldsymbol{x}, y) \approx 1$. Since both translation processes are clearly effective, it follows that, with the possible exception of the linear complexity claim, a result like that in Proposition 6.7 holds for $B A$ as well. It follows that the theory $B A$ of Boolean algebras is Gaussian.

Furthermore, the computational complexity of computing local solvers in $B A$ is indeed linear. This is thanks to the fact that local solvers in $B A$ can be computed directly, without a translation into the signature of $B R$. In fact, for each $e$-formula $u(\boldsymbol{x}, y) \approx 1$ (and fresh variable $z$ ), the term

$$
s^{\prime}(\boldsymbol{x}, z)=(u(\boldsymbol{x}, 1) \supset u(\boldsymbol{x}, z)) \supset(z \cap(u(\boldsymbol{x}, 0) \supset u(\boldsymbol{x}, z)))
$$

is a local solver for $u(\boldsymbol{x}, y) \approx 1$ in $B A$ w.r.t. $y$. It is immediate that $s^{\prime}(\boldsymbol{x}, z)$ can be computed in linear time from $u(\boldsymbol{x}, y)$. To see that it is indeed a local solver of $u(\boldsymbol{x}, y)$, one can argue as follows. From formulas (9) and (7), we have that 


$$
s(\boldsymbol{x}, z)=z+t(\boldsymbol{x}, z) *(1+t(\boldsymbol{x}, 1) * t(\boldsymbol{x}, 0)) *(z+1+t(\boldsymbol{x}, 1))
$$

is a local solver of the formula $t(\boldsymbol{x}, y) \approx 0$ for any $\Sigma_{B R^{-}}$term $t(\boldsymbol{x}, y)$. Observing that $u \approx 1$ is equivalent in $B A$ to $\bar{u} \approx 0$, let $t(\boldsymbol{x}, y)$ be the translation of $\bar{u}$ into the signature of $B R .{ }^{24}$ Then, modulo the signature translation, $t$ is equivalent to $\bar{u}$. Let $u_{z}, u_{0}, u_{1}$ abbreviate respectively $u(\boldsymbol{x}, z), u(\boldsymbol{x}, 0), u(\boldsymbol{x}, 1)$. If we replace every occurrence of $t(\boldsymbol{x}, z), t(\boldsymbol{x}, 0), t(\boldsymbol{x}, 1)$ in (12) by $\bar{u}_{z}, \bar{u}_{0}, \bar{u}_{1}$, respectively, and translate the formula (11) into the signature of $B R$, we obtain a formula that is equivalent in $B R$ to (12). To see that, consider the following chains of equalities modulo the signature translation and the axioms of $B A$ and $B R:{ }^{25}$

$$
\begin{aligned}
s^{\prime}(\boldsymbol{x}, z) & \approx\left(u_{1} \supset u_{z}\right) \supset\left(z \cap\left(u_{0} \supset u_{z}\right)\right) \\
& \approx \bar{u}_{1} \supset u_{z} \cup\left(z \cap\left(u_{0} \supset u_{z}\right)\right) \\
& \approx\left(u_{1} \cap \bar{u}_{z}\right) \cup\left(z \cap\left(\bar{u}_{0} \cup u_{z}\right)\right) \\
& \approx\left(u_{1} \bar{u}_{z}\right) \cup\left(z\left(\bar{u}_{0}+u_{z}+\bar{u}_{0} u_{z}\right)\right) \\
& \approx\left(u_{1} \bar{u}_{z}\right) \cup\left(z\left(1+u_{0}+u_{0} u_{z}\right)\right) \\
& \approx\left(u_{1} \bar{u}_{z}\right) \cup\left(z+u_{0} z+u_{0} u_{z} z\right) \\
& \approx u_{1} \bar{u}_{z}+z+u_{0} z+u_{0} u_{z} z+u_{1} \bar{u}_{z} z+u_{1} \bar{u}_{z} u_{0} z+u_{1} \bar{u}_{z} u_{0} u_{z} z \\
& \approx u_{1} \bar{u}_{z}+z+u_{0} z+u_{0} u_{z} z+u_{1} \bar{u}_{z} z+u_{0} u_{1} \bar{u}_{z} z \\
& \approx u_{1}+u_{1} u_{z}+z+u_{0} z+u_{0} u_{z} z+u_{1} z+u_{1} u_{z} z+u_{0} u_{1} z+u_{0} u_{1} u_{z} z, \\
s(\boldsymbol{x}, z) & \approx z+t(\boldsymbol{x}, z)(1+t(\boldsymbol{x}, 1) t(\boldsymbol{x}, 0))(z+1+t(\boldsymbol{x}, 1)) \\
& \approx z+\bar{u}_{z}\left(1+\bar{u}_{1} \bar{u}_{0}\right)\left(z+u_{1}\right) \\
& \approx z+\bar{u}_{z}\left(u_{1}+u_{0}+u_{0} u_{1}\right)\left(z+u_{1}\right) \\
& \approx z+\bar{u}_{z}\left(u_{1} z+u_{0} z+u_{0} u_{1} z+u_{1}+u_{0} u_{1}+u_{0} u_{1}\right) \\
& \approx z+\left(1+u_{z}\right)\left(u_{1} z+u_{0} z+u_{0} u_{1} z+u_{1}\right) \\
& \approx z+u_{1} z+u_{0} z+u_{0} u_{1} z+u_{1}+u_{1} u_{z} z+u_{0} u_{z} z+u_{0} u_{1} u_{z} z+u_{1} u_{z} .
\end{aligned}
$$

It is easy to verify at this point that both $s$ and $s^{\prime}$ reduce to the same $\Sigma_{B R^{-}}$ term, hence they are equivalent.

\subsection{Equational theories induced by modal logics}

Taken together, the results of the previous two subsections (Propositions 6.1, $6.2,6.3,6.7)$ show that the theory $B A$ is absolutely combinable.

$\overline{24}$ This translation can be achieved by the rewrite rules $\bar{x} \rightarrow x+1, x \cap y \rightarrow x * y$, and $x \cup y \rightarrow x+y+x * y$.

${ }^{25}$ To simplify the notation, we omit writing the operator $*$ explicitly, and use the standard precedence rules for $*$ and + . 
Theorem 6.8 Let $E_{1}, E_{2}$ be equational extensions of $B A$ having decidable word problems. Then the word problem in their union $E_{1} \cup E_{2}$ is also decidable.

Recall that an equational extension of $B A$ is an equational theory $E$ over a signature extending the signature of $B A$ and satisfying $\approx_{B A} \subseteq \approx_{E}$. Proposition 6.2 says that $\approx_{E}$ is in fact a conservative extension of $\approx_{B A}$ whenever $E$ is non-trivial. If one of the theories $E_{i}$ in the formulation of the above theorem is trivial, then the theorem holds trivially. Otherwise, we can apply Theorem 5.5.

It remains to show what all this has to do with modal logics and their fusions. In this subsection we show that there is a close connection between equational extensions of $B A$ and so-called classical modal logics, and that the union of such theories corresponds to the fusion of such modal logics.

A modal signature $\Sigma_{M}$ is a set of operation symbols endowed with corresponding arities; from $\Sigma_{M}$, propositional formulae are built using countably many propositional variables, the operation symbols in $\Sigma_{M}$, the Boolean connectives, and the constant $T$ for truth and $\perp$ for falsity. We use the letters $x, x_{1}, \ldots, y, y_{1}, \ldots$ to denote propositional variables and the letters $t, t_{1}, \ldots, u$, $u_{1}, \ldots$ to denote propositional formulae.

The following definition is taken from [33], pp. 8-9: ${ }^{26}$

Definition 6.9 A classical modal logic $L$ based on a modal signature $\Sigma_{M}$ is a set of propositional formulae that

(i) contains all classical propositional tautologies;

(ii) is closed under uniform substitution of propositional variables by propositional formulae;

(iii) is closed under the modus ponens rule ('from $t$ and $t \Rightarrow u$ infer $u$ ');

(iv) is closed under the replacement rules, which are specified as follows. We have one such rule for each $n$-ary o $\in \Sigma_{M}$, namely:

$$
\frac{t_{1} \Leftrightarrow u_{1}, \ldots, t_{n} \Leftrightarrow u_{n}}{o\left(t_{1}, \ldots, t_{n}\right) \Leftrightarrow o\left(u_{1}, \ldots, u_{n}\right)}
$$

Since classical modal logics (based on a given modal signature) are closed under intersections, it makes sense to speak of the least classical modal logic $[S]$ containing a certain set of propositional formulae $S$. If $L=[S]$, we say that $S$ is a set of axiom schemata for $L$ and write $S \vdash t$ for $t \in[S]$.

\footnotetext{
${ }^{26}$ Strictly speaking, K. Segerberg in [33] considers only modal signatures consisting of a single unary modal operator (i.e., unary unimodal logics; more general multimodal systems became popular only later on). The least classical modal logic with a single unary operator is usually called $\mathbf{E}$.
} 
Notice that giving a set of axiom schemata for $L$ is not the only way to introduce a classical modal logic $L$ : for instance, one can introduce $L$ just by specifying a certain (e.g. Kripke, neighborhood, algebraic, etc.) semantics and saying that $L$ is the set of formulae that are valid in that semantics.

We say that a classical modal logic $L$ is decidable iff $L$ is a recursive set of propositional formulae; the decision problem for $L$ is just the membership problem for $L$.

A classical modal logic $L$ is said to be normal iff for every $n$-ary modal operator $o$ in the signature of $L$ and every $i=1, \ldots, n, L$ contains the formula

$$
o\left(\boldsymbol{x}, \top, \boldsymbol{x}^{\prime}\right)
$$

and also the formula

$$
o\left(\boldsymbol{x},(y \Rightarrow z), \boldsymbol{x}^{\prime}\right) \Rightarrow\left(o\left(\boldsymbol{x}, y, \boldsymbol{x}^{\prime}\right) \Rightarrow o\left(\boldsymbol{x}, z, \boldsymbol{x}^{\prime}\right)\right)
$$

where $\boldsymbol{x}$ abbreviates the tuple $\left(x_{1}, \ldots, x_{i-1}\right)$ and $\boldsymbol{x}^{\prime}$ abbreviates the tuple $\left(x_{i+1}, \ldots, x_{n}\right)$. The latter schema is called the "Aristotle law". ${ }^{27}$ The least normal (classical modal, unary, unimodal) logic is the modal logic usually called K [34].

Most well-known modal logics considered in the literature (both normal and non-normal) fit Definition 6.9: these include standard unary unimodal systems like $\mathbf{K}, \mathbf{T}, \mathbf{K} 4, \mathbf{S 4}, \mathbf{S 5}$ and so on [34], tense systems like $\mathbf{K}_{t}$ and other temporal logics [35], the propositional dynamic logic $P D L$ [36], common knowledge systems [37], and computational tree logic CTL [38]. ${ }^{28}$ Modal logics with so-called graded modalities [40-42] (which correspond to qualified number restrictions in Description Logics [43]) are examples of classical modal logics that are not normal [17].

We want to show that any classical modal logic $L$ gives rise to an equational extension $E_{L}$ of $B A$ such that the decision problem for $L$ corresponds to the word problem for $E_{L}$. For notational convenience, we will assume that $\Sigma_{B A}$ also contains the binary symbol $\supset$, defined by the axiom $x \supset y \approx \bar{x} \cup y$.

Given a logic $L$ with modal signature $\Sigma_{M}$, we define $E_{L}$ as the theory having as signature $\Sigma_{M} \cup \Sigma_{B A}$ and as set of axioms the set

$$
B A \cup\left\{t_{B A} \approx 1 \mid t \in L\right\},
$$

$\overline{27}$ The axiom schema $o\left(\boldsymbol{x}, \top, \boldsymbol{x}^{\prime}\right)$ can be dropped by closing the logic under the necessitation rule: from $t$ infer $o\left(\boldsymbol{x}, t, \boldsymbol{x}^{\prime}\right)$; in that case, thanks to the Aristotle laws, the replacement rules become redundant.

${ }^{28}$ On the other hand, the full computational tree logic $C T L^{*}$ [39] is not a classical modal system in the sense of Definition 6.9 , as it is not closed under uniform substitution. 
where $t_{B A}$ is obtained from $t$ by replacing $t$ 's logical connectives $(\neg, \wedge, \vee, \Rightarrow)$ by the corresponding Boolean algebra operators $(\overline{(-)}, \cap, \cup, \supset)$, and the logical constants $\top$ and $\perp$ by 1 and 0 , respectively.

Vice versa, given an equational extension $E$ of $B A$ over the signature $\Sigma$, we define $L_{E}$ as the classical modal logic over the modal signature $\Sigma \backslash \Sigma_{B A}$ axiomatized by the formulae

$$
\left\{t_{L} \mid \models_{E} t \approx 1\right\}
$$

where $t_{L}$ is obtained from $t$ by the inverse of the replacement process above.

Classical modal logics (in our sense) and equational extensions of $B A$ are equivalent formalisms, as is well-known from algebraic logic [44]. In particular, for our purposes, the following standard proposition is crucial, as it reduces the decision problem for a classical modal logic $L$ to the word problem in $E_{L}$.

Proposition 6.10 For every classical modal logic $L$ and for every propositional formula $t$, we have that $t \in L$ iff $\left.\right|_{E_{L}} t_{B A} \approx 1$.

Proof. The direction from left to right is immediate from the definition of $E_{L}$.

For the opposite direction, we can use the well-known Lindenbaum algebra construction (see e.g. [44]). ${ }^{29}$ We define a model $\mathcal{A}_{L}$ of $E_{L}$ as follows. Where $\Sigma_{L}$ is the signature of $L$, the carrier of $\mathcal{A}_{L}$ is defined as the set of all the equivalence classes of $\Sigma_{L}$-formulae with respect to the equivalence relation ${ }^{30}$

$$
\equiv:=\{(t, u) \mid t \Leftrightarrow u \in L\}
$$

It is easy to see that, since $L$ is closed under the replacement rules, $\equiv$ is in fact a congruence relation with respect to the modal operators in $\Sigma_{L}$. We define these operators in $\mathcal{A}_{L}$ as prescribed by $L$, that is, we interpret each $n$-ary modal operator $o$ as the $n$-ary function $o^{\mathcal{A}_{L}}$ such that

$$
o^{\mathcal{A}_{L}}\left(\left[t_{1}\right]_{\equiv}, \ldots,\left[t_{n}\right]_{\equiv}\right)=\left[o\left(t_{1}, \ldots, t_{n}\right)\right]_{\equiv} .
$$

We then define the Boolean operators in the obvious way, that is, we interpret $\cap$, say, as the binary function $\cap^{\mathcal{A}_{L}}$ such that $\cap \mathcal{A}_{L}\left(\left[t_{1}\right]_{\equiv},\left[t_{2}\right]_{\equiv}\right)=\left[t_{1} \wedge t_{2}\right]_{\equiv}$, and so on. It is a standard exercise to show that $\mathcal{A}_{L}$ is well-defined. From the closure of $L$ under uniform substitution, we obtain for arbitrary formulae $t, u$ that $\mathcal{A}_{L}=t_{B A} \approx u_{B A}$ iff $t \Leftrightarrow u \in L$; for $u=1$, we also get (by modus ponens

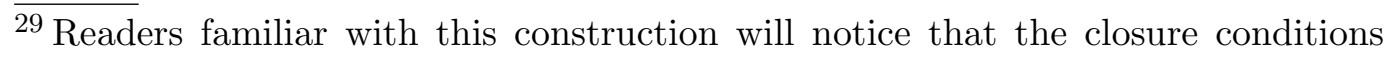
required by Definition 6.9 are precisely the closure conditions that make the construction work.

${ }^{30}$ That $\equiv$ is in fact an equivalence relation follows from modus ponens and tautologies.
} 
and tautologies) that $\mathcal{A}_{L}=t_{B A} \approx 1$ iff $t \in L$. This shows, in particular, that $\mathcal{A}_{L}$ is a model of the equational theory $E_{L}$. Hence if $\models_{E_{L}} t_{B A} \approx 1$, we have in particular that $\mathcal{A}_{L} \models t_{B A} \approx 1$ and finally that $t \in L$, as claimed.

Given two classical modal logics $L_{1}, L_{2}$ over two disjoint modal signatures $\Sigma_{M}^{1}, \Sigma_{M}^{2}$, the fusion of $L_{1}$ and $L_{2}$ is the classical modal logic

$$
L_{1} \otimes L_{2}
$$

over the signature $\Sigma_{M}^{1} \cup \Sigma_{M}^{2}$ defined as $\left[L_{1} \cup L_{2}\right]$, the least classical modal logic extending $L_{1} \cup L_{2}$. Since $E_{L_{1} \otimes L_{2}}$ is easily seen to be deductively equivalent to the theory $E_{L_{1}} \cup E_{L_{2}}$ (i.e., $\approx_{E_{L_{1} \otimes L_{2}}}=\approx_{E_{L_{1}} \cup E_{L_{2}}}$ ), it is clear that the decision problem $L_{1} \cup L_{2} \vdash t$ reduces to the word problem $E_{L_{1}} \cup E_{L_{2}}=t_{B A} \approx 1$. Theorem 6.8 thus yields the following transfer theorem for classical modal logics.

Theorem 6.11 If $L_{1}, L_{2}$ are decidable classical modal logics, then their fusion $L_{1} \otimes L_{2}$ is also decidable.

\subsection{Complexity issues}

The complexity of our combination procedure applied to fusion decidability in modal logic is the same as the complexity of the combination procedures proposed for the classical normal modal logics case in [15] and for the classical modal logics with covering normal terms treated in [17]. In fact, the same remarks as in [17] apply, as we will see below.

To begin with, let us recall that

- the preprocessing abstraction procedure ${ }^{31}$ takes only linear time;

- the computation of a local solver takes also linear time - although it might be applied to an exponentially long formula, as we will see;

- only linearly many iterations of our procedure's loop (see Fig. 1) need to be executed on any input.

Consequently, the only sources of real complexity in the whole procedure are the tests of Step 3 of the loop (the final test, after the loop, is of the same nature). Hence we have to analyze:

- how many such tests are performed;

- how expensive each of them is.

$\overline{31}$ The one that converts a formula in the signature of the fusion logic into an initial ARS and two Boolean terms $u$ and $v$. 
Suppose that $n$ is the number of the free constants in the procedure's inputthe initial ARS $R$ and the shared terms $u$ and $v$. This number is obviously linear in the size of the input. Let us assume for simplicity that the only free constants in the input are the lhs constants in $R: a_{1}, \ldots, a_{n}{ }^{32}$ Now, as we discussed before stating Proposition 6.1, the number of non-equivalent Boolean terms over $n$ constants is $2^{2^{n}}$, hence one might conclude that during the $i^{\text {th }}$ iteration of the procedure's loop we will need to execute $O\left(2^{2^{i}} \cdot 2^{2^{i}}\right)$ equivalence tests in Step 3 of the loop. Instead, we can limit ourselves to $2^{i}$ tests for the following reason.

Recall that the $e$-formula $\varphi$ built at Step 4 of the loop is equivalent in the shared theory $B A$ to an identity of the form $t \approx 1$, where $t$ is a Boolean term. This term is in turn equivalent in $B A$ to a term of the form $t_{1} \cap \cdots \cap t_{m}$, where each $t_{k}$ is a term-clause, i.e., a term of the form $b_{1} \cup \cdots \cup b_{i}$ where each $b_{j}$ is either $a_{j}$ or $\bar{a}_{j}$. It is an immediate consequence of $B A$ that

$$
\models_{B A}\left(t_{1} \cap \cdots \cap t_{m}\right) \approx 1 \text { iff } \models_{B A} t_{k} \approx 1 \text { for all } k=1, \ldots, m .
$$

It follows that to generate $\varphi$ it is enough to consider in the test of Step 3 only identities of the form $t \approx 1$ where $t$ is a term-clause over $a_{1}, \ldots, a_{i}$. And we already know that, modulo $B A$, there are only $2^{i}$ such identities. As an additional consequence of the above we have that the size of the $e$-formula $\varphi$ is linear in $2^{i}$, which in turn means that the local solver computed in Step 6 of the loop is also linear in $2^{i}$, and so exponential in the size of the input.

Let us now consider the cost of the test

$$
R_{j}^{(i)} \models E_{L_{j}} t \approx 1
$$

where $t$ is any term-clause. This test requires $R_{j}^{(i)}$-normalization first and then a call to the decision procedure for the input logic $L_{j}$. In the worst case, $R_{j}^{(i)}$ is of the form $\left\{a_{1} \rightarrow t_{1}, \ldots, a_{i} \rightarrow t_{i}\right\}$ with each right-hand side term being a recursively computed, exponentially long solver.

Normalizing the term $t$ with respect to $R_{j}^{(i)}$ can then raise the length of $t$ from linear to $2^{q(n)}$, where $q(n)$ is a quadratic polynomial. To see this it is helpful to observe that, because of the way $R_{j}^{(i)}$ is defined, normalizing $t$ amounts to first replacing every occurrence of $a_{1}$ in $t$ by $t_{1}$, then replacing every occurrence of $a_{2}$ in the resulting term by $t_{2}$ and so on. Now let us first consider how the size of the terms $t_{1}, \ldots, t_{i}$ grows when we apply the rewrite system to them. First of all, $t_{1}$ is irreducible, and so it does not change in size, i.e., its size after rewriting is still $O\left(2^{n}\right)$. The term $t_{2}$ is of size $O\left(2^{n}\right)$ and thus may contain at most $O\left(2^{n}\right)$ occurrences of $a_{1}$. Thus, by rewriting, its size may grow to $O\left(2^{n}+2^{n} \cdot 2^{n}\right)=O\left(2^{2 n}\right)$. The term $t_{3}$ is of size $O\left(2^{n}\right)$ and thus may contain at

\footnotetext{
$\overline{32}$ The complexity analysis does not change if we ignore other possible free constants.
} 
most $O\left(2^{n}\right)$ occurrences of $a_{1}, a_{2}$. Considering the worst-case that all of them are occurrences of $a_{2}$, the size of $t_{3}$ may grow to $O\left(2^{n}+2^{n} \cdot 2^{2 n}\right)=O\left(2^{3 n}\right)$. If we continue this argument until we reach $t_{n}$, we see that indeed $t_{n}$ may grow by rewriting to size $O\left(2^{\left(n^{2}\right)}\right)$. Since the size of the term $t$ is linear in $n,{ }^{33}$ its size may grow by rewriting (where in the worst case we replace $O(n)$ constants by terms of size $\left.O\left(2^{\left(n^{2}\right)}\right)\right)$ to size $O\left(2^{\left(n^{2}+1\right)}\right)$.

In conclusion, the decision procedures for $L_{1}$ and for $L_{2}$ may have to deal with exponentially many, exponentially long instances of the decision problem in each of the linearly many iterations of the loop. If these procedures are in PSPACE, we get an EXPSPACE combined decision procedure. If instead the procedures are in EXPTIME, we get a 2EXPTIME combined decision procedure. These are the same as the complexity bounds given in [17] for their combination procedure.

\subsection{Examples}

Here we give two examples of our combination procedure at work in the case of classical modal logics.

Example 6.12 Consider the classical modal logic KT with modal signature $\{\square\}$ and obtained by adding to $\mathbf{K}$ the axiom schema

$$
\square x \Rightarrow x
$$

Now let $\mathbf{K} \mathbf{T}_{1}$ and $\mathbf{K} \mathbf{T}_{2}$ be two signature disjoint renamings of $\mathbf{K T}$ in which $\square_{1}$ and $\square_{2}$, respectively, replace $\square$, and consider the fusion logic $\mathbf{K T}_{1} \otimes \mathbf{K} \mathbf{T}_{2}$. We can use our combination procedure to show that

$$
\mathbf{K} \mathbf{T}_{1} \otimes \mathbf{K T}_{2} \vdash \square_{2} x \Rightarrow \diamond_{1} x
$$

(where as usual $\diamond_{1} x$ abbreviates $\neg \square_{1} \neg x$ ).

For $i=1,2$, let $E_{i}$ be the equational theory corresponding to $\mathbf{K T}_{i}$. It is enough to show that

$$
\models_{E_{1} \cup E_{2}}\left(\square_{2}(x) \supset \diamond_{1}(x)\right) \approx 1
$$

where now $\diamond_{1} x$ abbreviates $\overline{\square_{1}(\bar{x})}$.

After the abstraction process, we get the two rewrite systems:

$$
R_{1}=\left\{a_{1} \rightarrow \diamond_{1}(c)\right\} \text { and } R_{2}=\left\{a_{2} \rightarrow \square_{2}(c)\right\}
$$

and the goal equation

$$
\left(a_{2} \supset a_{1}\right) \approx 1
$$

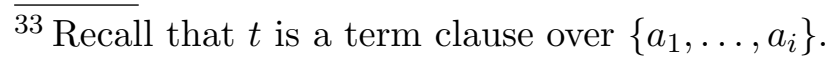


where $a_{1}, a_{2}$ and $c$ are fresh constants.

Recall from our discussion in Section 6.4 that for the test in Step 3 of the procedure's loop we need to consider only identities of the form $t \approx 1$ where $t$ is a term-clause over the set of constants under consideration. During the first execution of the procedure's loop the constants in question are $a_{1}$ and $c$; therefore there are only four identities to consider:

$$
\bar{a}_{1} \cup \bar{c} \approx 1, \quad \bar{a}_{1} \cup c \approx 1, \quad a_{1} \cup \bar{c} \approx 1, a_{1} \cup c \approx 1
$$

The only identity for which the test in Step 3 is positive is $a_{1} \cup \bar{c}$. In fact, $a_{1} \cup \bar{c}$ rewrites to $\diamond_{1}(c) \cup \bar{c}$, which is equivalent to $c \supset \diamond_{1}(c)$. This is basically the contrapositive of (the translation of) the axiom schema $\square_{1}(c) \supset c$. ${ }^{34}$

Using the formula

$$
s(\boldsymbol{x}, z)=(u(\boldsymbol{x}, 1) \supset u(\boldsymbol{x}, z)) \supset(z \cap(u(\boldsymbol{x}, 0) \supset u(\boldsymbol{x}, z)))
$$

from Subsection 6.2, we can produce a solver for that identity, which reduces to $c \cup d_{1}$ after some simplifications, where $d_{1}$ is a fresh free constant. Hence, the following rewrite rule is added to $R_{2}$ in Step 6 of the loop:

$$
a_{1} \rightarrow c \cup d_{1} .
$$

Note that at this time we could already quit the loop and provide an output using $R_{2}$ and $E_{2}$ in the final test instead of $R_{1}$ and $E_{1} .{ }^{35}$ If we did that, the final test $R_{2} \models_{E_{2}}\left(a_{2} \supset a_{1}\right) \approx 1$ (that is, $\models_{E_{2}} \square_{2}(c) \supset\left(c \cup d_{1}\right) \approx 1$ ) would succeed because the corresponding modal formula

$$
\square_{2} c \Rightarrow\left(c \vee d_{1}\right)
$$

is in fact a theorem of $\mathbf{K} \mathbf{T}_{2}$.

Continuing the execution of the loop with the second - and final-iteration, we get instead the following. Among the eight term-clauses involving $a_{1}, a_{2}, c$, the test in Step 3 is positive for four of them. The conjunction of such termclauses gives a Boolean $e$-formula that is equivalent to $\left(a_{2} \supset c\right) \cap\left(c \supset a_{1}\right) \approx 1$. This $e$-formula, once solved with respect to $a_{2}$, gives (after simplifications) the rewrite rule

$$
a_{2} \rightarrow d_{2} \cap\left(\left(c \supset a_{1}\right) \supset\left(d_{2} \supset c\right)\right),
$$

\footnotetext{
${ }^{34}$ Another approach for checking this, and also that the tests for the other termclauses are negative, is to translate the rewritten term-clauses into the corresponding modal formulae, and then check whether their complement is unsatisfiable in all Kripke structures with a reflexive accessibility relation (see [45], Fig. 5.1).

${ }^{35}$ Recall that it is immaterial whether $R_{1}$ and $E_{1}$ or $R_{2}$ and $E_{2}$ are used for the final test.
} 
which is added to $R_{1}$ before quitting the loop. Using this $R_{1}$, the final test of the procedure $\left(R_{1} \models E_{E_{1}} a_{2} \supset a_{1} \approx 1\right)$ succeeds because the modal formula

$$
d_{2} \wedge\left(\left(c \Rightarrow \diamond_{1} c\right) \Rightarrow\left(d_{2} \Rightarrow c\right)\right) \Rightarrow \diamond_{1} c
$$

is a theorem of $\mathbf{K} \mathbf{T}_{1}$.

Example 6.13 Here we consider the fusion $\mathbf{R} \otimes \mathbf{K T B}$, where $\mathrm{KTB}$ is the classical modal logic obtained by adding to $\mathbf{K T}$ the axiom schema

$$
\diamond \square x \Rightarrow x
$$

and $\mathbf{R}$ is obtained from the minimum classical unimodal system $\mathbf{E}$, with modal operator $\mathrm{O}$, by adding to it the regularity rule: ${ }^{36}$

$$
\frac{t \Rightarrow u}{\mathrm{o} t \Rightarrow \mathrm{o} u}
$$

Note that $\mathbf{R}$ is classical, but not normal. We consider the fusion $\mathbf{R} \otimes \mathbf{K T B}$. In the combination procedure, we must test term clauses for validity in $\mathbf{R}$ and in KTB. For KTB, this can be achieved, for instance, by checking the complement of the modal formulae obtained after rewriting for unsatisfiability in all Kripke structures with a reflexive and symmetric accessibility relation (see again [45], Figure 5.1). For $\mathbf{R}$, one can check, for instance, the complement of the modal formulae obtained after rewriting for unsatisfiability in all neighborhood frames where the set of sets of worlds associated with each world is closed under supersets (see, e.g., [33], page 43).

We apply our combined procedure to show that

$$
\mathbf{R} \otimes \mathbf{K T B} \vdash \diamond \square \circ x \Rightarrow \circ \diamond x
$$

After purification, we obtain the ARS consisting of

$$
R_{1}=\left\{a_{4} \rightarrow \circ a_{1}, a_{2} \rightarrow \circ c\right\} \quad \text { and } \quad R_{2}=\left\{a_{1} \rightarrow \diamond c, a_{3} \rightarrow \diamond \square a_{2}\right\}
$$

and the goal identity

$$
\left(a_{3} \supset a_{4}\right) \approx 1
$$

In the first iteration of the loop, we test the term-clauses over $a_{1}, c$, and get $\left(a_{1} \cup \bar{c}\right) \approx 1$ as the $e$-formula to be solved with respect to $a_{1}$. As in the first step of the previous example, the solver (after simplifications) gives the rewrite rule $a_{1} \rightarrow\left(c \cup d_{1}\right)$.

\footnotetext{
${ }^{36}$ Instead of the regularity rule, one may equivalently use the axiom schema $\bigcirc(t \wedge$ $u) \Rightarrow$ O $u$ to get the logic $\mathbf{R}$ (see [33], page 45).
} 
In the second iteration, nothing relevant happens because the $e$-formula to be solved with respect to $a_{2}$ is equivalent to an $e$-formula (namely $\left(a_{1} \cup \bar{c}\right) \approx 1$ again) in which $a_{2}$ does not occur. This entails that using (14) to compute the local solver yields the trivial rewrite rule $a_{2} \rightarrow d_{2}$ for some fresh constant $d_{2}$. In the third iteration, term-clauses involving $a_{1}, a_{2}, a_{3}, c$ are tested; this results in an $e$-formula equivalent to $\left(a_{3} \supset a_{2}\right) \cap\left(c \supset a_{1}\right) \approx 1$. Solving it with respect to $a_{3}$ gives (after simplifications) the rule $a_{3} \rightarrow d_{3} \cap\left(\left(c \supset a_{1}\right) \supset\left(d_{3} \supset a_{2}\right)\right)$.

We can ignore the last iteration of the loop because it modifies $R_{2}$, which is not used afterwards. Performing the final test using $R_{1}$, the modal formula to be tested for validity in $\mathbf{R}$ is then

$$
\left(d_{3} \wedge\left(\left(c \Rightarrow\left(c \vee d_{1}\right)\right) \Rightarrow\left(d_{3} \Rightarrow \circ c\right)\right)\right) \Rightarrow \circ\left(c \vee d_{1}\right)
$$

This formula is indeed valid in $\mathbf{R}$. To see that, first notice that the subformula $c \Rightarrow\left(c \vee d_{1}\right)$ is a tautology. Therefore it is enough to show the validity of

$$
\left(d_{3} \wedge\left(d_{3} \Rightarrow \circ c\right)\right) \Rightarrow \circ\left(c \vee d_{1}\right)
$$

This follows from the transitivity of implication, because $\left(d_{3} \wedge\left(d_{3} \Rightarrow \circ c\right)\right) \Rightarrow \circ c$ and $\bigcirc c \Rightarrow \odot\left(c \vee d_{1}\right)$ are both valid in $\mathbf{R}$ (for the latter, apply the regularity rule to the tautology $\left.c \Rightarrow\left(c \vee d_{1}\right)\right)$.

As a final remark observe that if we replace in the example the logic $\mathbf{R}$ by the logic $\mathbf{E}$, the execution of the procedure is the same but the final test is negative. To get a falsifying model for the modal propositional formula in the final test, it is sufficient to observe that any Boolean algebra in which the operator $O$ is interpreted as the Boolean complement is a model of E. ${ }^{37}$

\section{Conclusion}

In this paper, we have described a new approach for combining decision procedures for the word problem in equational theories over non-disjoint signatures. Unlike the previous combination methods for the word problem in the nondisjoint case $[10,11]$, this approach has the known decidability transfer results for validity in the fusion of modal logics [12,15] as consequences. Our combination result is, however, more general than these transfer results since it applies also to non-normal modal logics - thus answering in the affirmative a long-standing open question in modal logics - and to equational theories not induced by modal logics (see, e.g., Example 3.6). Despite the generality of our

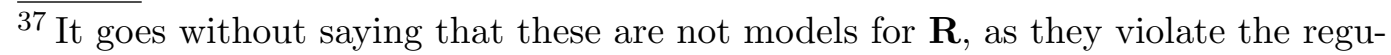
larity rule. 
approach, for the modal logic application the complexity upper-bounds we obtain are the same as for the existing, more restricted approaches $[15,17]$.

Our results are not consequences of combination results for the conditional word problem (the relativized validity problem) recently obtained by generalizing the Nelson-Oppen combination method $[19,20]$. In fact, there are modal logics for which the validity problem is decidable, but the relativized validity problem is not. This is, e.g, the case for the modal logic obtained from the product of the modal logic $\mathbf{K}$ with itself [18], and for modal logics obtained by translating certain description logics into modal logic notation, such as description logics with feature agreements [46] or with concrete domains [47].

Our new combination approach is orthogonal to the previous combination approaches for the word problem in equational theories over non-disjoint signature $[10,11]$. On the one hand, the previous results do not apply to theories induced by modal logics [11]. On the other hand, there are equational theories that satisfy the restrictions imposed by the previous approaches, but are not locally finite [10], and thus do not satisfy our restrictions. Both the approach described in this paper and those in $[10,11]$ have the known combination results for the case of disjoint signatures as a consequence. For the previous approaches, this was already pointed out in $[10,11]$. For our approach, this is an immediate consequence of the fact that the pure equality theory $E_{=}$(see Example 3.7) is absolutely combinable.

Compared to the compatibility condition introduced in a preliminary version of this work [48], the one defined here is less restrictive (i.e., it applies to more theories). Whereas in [48] we required that every model of $E$ embeds into a model of $E \cup E_{0}^{*}$, Definition 3.1 only requires this for every finitely generated free model of $E$. In our examples, this greatly simplifies proving that the compatibility condition is satisfied.

One direction for future research could be to check whether the algebraic approach employed in this paper can also be used to obtain transfer results for other interesting properties of modal logics, such as interpolation. Another direction could be to find absolutely combinable theories other than the ones considered here. In the context of modal logics, it would be interesting to find cases in which decidability transfers even if the component theories share a theory strictly extending the theory of Boolean algebras. A good candidate for such a shared theory might be the equational theory $E_{\mathbf{S} 5}$ induced by the modal logic S5 since it is locally finite. Working with S5, however, would require substantial modifications to our combination procedure because $E_{\mathbf{S 5}}$ is not Gaussian.

Further research could also go in the direction of enhancing our procedure to improve its performance in practice. The current formulation of the procedure 
privileges simplicity of exposition over efficiency. Several efficiency improvements are however conceivable. For instance, it is clear from the definition of local solver that when computing the formula $\varphi\left(\boldsymbol{a}_{i}, \boldsymbol{c}\right)$ in Steps 3 and 4 of the main loop it is enough to consider only those identities that contain the constant $a_{i}$-and so ignore, in particular, the identities already considered in previous steps. Also, it should be possible to remove the totality requirement on the ordering $>$ of the left-hand side constants in the input ARS $R$ without affecting the correctness of the procedure. It should be enough to consider the smallest partial ordering $>$ such that for all $a \rightarrow t \in R$, the constant $a$ is greater than all left-hand side constants in $t$. The net effect of this relaxation is that the rewrite system and the tuple of constants considered in each iteration of the loop would often be smaller, reducing again the number of identities to consider in Step 3. Additional efficiency in computing $\varphi\left(\boldsymbol{a}_{i}, \boldsymbol{c}\right)$ can come from using theory specific information as well. We have already seen an example of this in Subsection 6.4, where we argue that for the theory of Boolean algebras it is enough to consider only identities of the form $t \approx 1$ with $t$ a term-clause over the relevant constants. This reduces the number of identities to be considered from double-exponential to single-exponential.

\section{Acknowledgments}

We thank the anonymous reviewers for their helpful comments on how to further improve the presentation of this paper.

\section{References}

[1] G. Nelson, D. C. Oppen, Simplification by cooperating decision procedures, ACM Trans. on Programming Languages and Systems 1 (2) (1979) 245-257.

[2] G. Nelson, Combining satisfiability procedures by equality-sharing, in: W. W. Bledsoe, D. W. Loveland (Eds.), Automated Theorem Proving: After 25 Years, Vol. 29 of Contemporary Mathematics, American Mathematical Society, Providence, RI, 1984, pp. 201-211.

[3] D. Pigozzi, The join of equational theories, Colloquium Mathematicum 30 (1) (1974) 15-25.

[4] E. Tidén, First-Order Unification in Combinations of Equational Theories, $\mathrm{PhD}$ dissertation, The Royal Institute of Technology, Stockholm (1986).

[5] M. Schmidt-Schauß, Unification in a combination of arbitrary disjoint equational theories, Journal of Symbolic Computation 8 (1-2) (1989) 51-100, special issue on unification. Part II. 
[6] T. Nipkow, Combining matching algorithms: The regular case, Journal of Symbolic Computation 12 (1991) 633-653.

[7] F. Baader, C. Tinelli, A new approach for combining decision procedures for the word problem, and its connection to the Nelson-Oppen combination method, in: W. McCune (Ed.), Proceedings of the 14th International Conference on Automated Deduction (Townsville, Australia), Vol. 1249 of Lecture Notes in Artificial Intelligence, Springer-Verlag, 1997, pp. 19-33.

[8] E. Domenjoud, F. Klay, C. Ringeissen, Combination techniques for non-disjoint equational theories, in: A. Bundy (Ed.), Proceedings of the 12th International Conference on Automated Deduction, Nancy (France), Vol. 814 of Lecture Notes in Artificial Intelligence, Springer-Verlag, 1994, pp. 267-281.

[9] C. Tinelli, C. Ringeissen, Unions of non-disjoint theories and combinations of satisfiability procedures, Theoretical Computer Science 290 (1) (2003) 291-353.

[10] F. Baader, C. Tinelli, Deciding the word problem in the union of equational theories, Information and Computation 178 (2) (2002) 346-390.

[11] C. Fiorentini, S. Ghilardi, Combining word problems through rewriting in categories with products, Theoretical Computer Science 294 (2003) 103-149.

[12] M. Kracht, F. Wolter, Properties of independently axiomatizable bimodal logics, The Journal of Symbolic Logic 56 (4) (1991) 1469-1485.

[13] E. Spaan, Complexity of modal logics, Ph.D. thesis, Department of Mathematics and Computer Science, University of Amsterdam, The Netherlands (1993).

[14] K. Fine, G. Schurz, Transfer theorems for stratified modal logics, in: J. Copeland (Ed.), Logic and Reality: Essays in Pure and Applied Logic. In Memory of Arthur Prior, Oxford University Press, 1996, pp. 169-213.

[15] F. Wolter, Fusions of modal logics revisited, in: M. Kracht, M. de Rijke, H. Wansing, M. Zakharyaschev (Eds.), Advances in Modal Logic, CSLI, Stanford, CA, 1998.

[16] D. M. Gabbay, Fibring Logics, Vol. 38 of Oxford Logic Guides, Clarendon Press, Oxford, 1999.

[17] F. Baader, C. Lutz, H. Sturm, F. Wolter, Fusions of description logics and abstract description systems, Journal of Artificial Intelligence Research 16 (2002) 1-58.

[18] D. M. Gabbay, A. Kurucz, F. Wolter, M. Zakharyaschev, Many-Dimensional Modal Logics: Theory and Applications, Elsevier, 2003.

[19] S. Ghilardi, L. Santocanale, Algebraic and model theoretic techniques for fusion decidability in modal logics, in: M. Vardi, A. Voronkov (Eds.), Proceedings of the 10th International Conference on Logic for Programming, Artificial Intelligence, and Reasoning (LPAR 2003), Vol. 2850 of Lecture Notes in Computer Science, Springer-Verlag, 2003, pp. 152-166. 
[20] S. Ghilardi, Model-theoretic methods in combined constraint satisfiability, Journal of Automated Reasoning 33 (3-4) (2004) 221-249.

[21] U. Martin, T. Nipkow, Boolean unification-The story so far, J. Symbolic Computation $7(3,4)(1989)$ 275-293.

[22] F. Baader, T. Nipkow, Term Rewriting and All That, Cambridge University Press, United Kingdom, 1998.

[23] M. A. Arbib, E. G. Manes, Arrows, Structures and Functors, Academic Press, 1975.

[24] S. McLane, Categories for the working mathematician, Springer-Verlag, 1971.

[25] G. Grätzer, Universal Algebra, 2nd Edition, Springer-Verlag, 1979.

[26] W. Hodges, Model Theory, Vol. 42 of Enclyclopedia of mathematics and its applications, Cambridge University Press, 1993.

[27] C.-C. Chang, H. J. Keisler, Model Theory, IIIrd Edition, North-Holland, Amsterdam-London, 1990.

[28] F. Baader, W. Snyder, Unification theory, in: J. Robinson, A. Voronkov (Eds.), Handbook of Automated Reasoning, Vol. I, Elsevier Science Publishers, 2001, pp. $447-533$.

[29] S. MacLane, G. Birckhoff, Algebra, MacMillan, New York, 1965.

[30] S. Ghilardi, M. Zawadowski, Sheaves, Games and Model Completions, Vol. 14 of Trends in Logic, Kluwer Academic Publishers, 2002.

[31] S. K. Thomason, Independent propositional modal logics, Studia Logica 39 (1980) 143-144.

[32] R. Balbes, P. Dwinger, Distributive Lattices, University of Missouri Press, 1974.

[33] K. Segerberg, An Essay in Classical Modal Logic, Vol. 13 of Filosofiska Studier, Uppsala Universitet, 1971.

[34] A. Chagrov, M. Zakharyaschev, Modal Logic, Vol. 35 of Oxford Logic Guides, Clarendon Press, Oxford, 1997.

[35] D. M. Gabbay, I. Hodkinson, M. Reynolds, Temporal Logic, Vol.I, Vol. 28 of Oxford Logic Guides, Clarendon Press, Oxford, 1994.

[36] V. Pratt, Semantical considerations on Floyd-Hoare logic, in: Proceedings of the 17th Annual Symposium on Foundations of Computer Science,, IEEE Comp. Soc., 1976, pp. 109-121.

[37] J. Y. Halpern, Y. Moses, A guide to completeness and complexity for modal logics of knowledge and belief, Artificial Intelligence 54 (3) (1992) 319-379.

[38] E. M. Clarke, A. E. Emerson, Design and synthesis of synchronisation skeletons usin branching time temporal logic, in: Logic of Programs,, Vol. 131, Lecture Notes in Computer Science, Springer-Verlag, 1982, pp. 52-71. 
[39] A. E. Emerson, J. Y. Halpern, 'sometimes' and 'not never' revisited: On branching versus linear time temporal logic, Journal of the ACM 33 (1) (1986) $151-178$.

[40] M. Fattorosi-Barnaba, F. De Caro, Graded modalities I, Studia Logica 44 (1985) $197-221$.

[41] W. Van der Hoek, M. de Rijke, Counting objects, J. Logic and Computation 5 (3) (1995) 325-345.

[42] S. Tobies, A PSPACE algorithm for graded modal logic, in: H. Ganzinger (Ed.), Proc. of the 16th Int. Conf. on Automated Deduction (CADE'99), Vol. 1632 of Lecture Notes in Artificial Intelligence, Springer-Verlag, 1999, pp. 52-66.

[43] B. Hollunder, F. Baader, Qualifying number restrictions in concept languages, in: Proc. of the 2nd Int. Conf. on the Principles of Knowledge Representation and Reasoning (KR'91), 1991, pp. 335-346.

[44] H. Rasiowa, An Algebraic Approach to Non-Classical Logics, Vol. 78 of Studies in Logic and the Foundations of Mathematics, North Holland, Amsterdam, 1974 .

[45] B. F. Chellas, Modal Logic, an Introduction, Cambridge University Press, Cambridge, 1980.

[46] F. Baader, H.-J. Bürckert, B. Nebel, W. Nutt, G. Smolka, On the expressivity of feature logics with negation, functional uncertainty, and sort equations, J. of Logic, Language and Information 2 (1993) 1-18.

[47] F. Baader, P. Hanschke, Extensions of concept languages for a mechanical engineering application, in: Proc. of the 16th German Workshop on Artificial Intelligence (GWAI'92), Vol. 671 of Lecture Notes in Computer Science, Springer-Verlag, Bonn (Germany), 1992, pp. 132-143.

[48] F. Baader, S. Ghilardi, C. Tinelli, A new combination procedure for the word problem that generalizes fusion decidability results in modal logics, in: Proceedings of the Second International Joint Conference on Automated Reasoning (IJCAR'04), Vol. 3097 of Lecture Notes in Artificial Intelligence, Springer-Verlag, Cork (Ireland), 2004, pp. 183-197. 